\title{
Diversidad de mariposas y su relación con el paisaje en la cuenca del río Lagunillas, Tolima, Colombia
}

\author{
Leonardo A. Ospina-López ${ }^{1,2, *}$, M. Gonzalo Andrade-C. ${ }^{1}$, Gladys Reinoso-Flórez² \\ ${ }^{1}$ Instituto de Ciencias Naturales - ICN, Universidad Nacional de Colombia, Bogotá, Colombia \\ ${ }^{2}$ Grupo de Investigación en Zoología, Facultad de Ciencias, Universidad del Tolima, Ibagué, Colombia
}

\begin{abstract}
Resumen
El presente estudio se desarrolló al norte del departamento del Tolima con el objetivo de caracterizar las mariposas asociadas a fragmentos de bosque, matorral o rastrojo y pastos. Se registraron las coordenadas geográficas de los bordes de los hábitats encontrados en una parcela de 0,6 km de radio y se establecieron las correlaciones entre los atributos del paisaje y la comunidad de mariposas, representada por seis familias y 266 especies. Las diferencias estructurales entre los hábitats de estudio pueden ayudar a explicar las diferencias en la riqueza y la diversidad de mariposas debido a que las comunidades responden diferencialmente al hábitat.
\end{abstract}

Palabras clave: mariposas, ecología, bosques, rastrojos, pastos.

Butterflies diversity and their relationship to the landscape in the Lagunillas River basin in north Tolima, Colombia

\begin{abstract}
The present study was conducted in north Tolima to characterize the butterflies associated with fragments of forest, brushwood/stubbles and grass. The geographical coordinates of the edges of every habitat in a smallholding of 0.6 $\mathrm{km}$ of radius were registered with the aim of finding the correlations between landscape attributes and the butterfly community represented by 266 species distributed in six families. Structural differences between the habitats can help explain the differences in the richness and diversity of butterflies because the communities respond differently to the habitat.
\end{abstract}

Key words: Butterflies, ecology, forest, stubble, pastures, Tolima.

\section{Introducción}

La transformación de los hábitats naturales y la variación de sus condiciones físicas pueden tener efectos negativos en algunas especies de animales y plantas. Una de las principales causas de la pérdida global de diversidad es la transformación de los bosques tropicales debido a las actividades humanas (Kattan, 2002) resultado de las políticas de estímulo a la ocupación y el uso del territorio, de la sobreexplotación, la fragmentación de las poblaciones, la deforestación, el consumo de leña, los incendios, la actividad agrícola, la contaminación, la introducción de especies, la pesca comercial sin control, la urbanización, la minería, la destrucción de humedales y zonas de páramo, la erosión, los desastres naturales y la cosecha indiscriminada; como causas indirectas cabe mencionar el desconocimiento del potencial estratégico de la biodiversidad, la débil capacidad institucional para reducir el impacto de las actividades que generan pérdida de biodiversidad, la expansión de la frontera agropecuaria, la baja presencia del Estado en las zonas de alta biodiversidad, el surgimiento y consolidación de los cultivos ilícitos, los problemas de orden público, los conflictos armados, el comercio internacional de pieles, la colonización y el desarrollo de proyectos de infraestructura (Andrade-C, 2011), lo que ha llevado a que los fragmentos de bosque queden inmersos en matrices modificadas, las cuales ofrecen diferentes calidades a la fauna.

Los hábitats que componen la matriz adyacente pueden llegar a contener una diversidad propia significativa que interactúa con los fragmentos de bosque (Gascon, et al., 1999, Kattan, 2002); de esta manera, las interacciones entre los elementos del paisaje pueden incidir en la diversidad y abundancia de las especies (Kattan, 2002), y permiten el movimiento de los organismos a través del mosaico de parches en el paisaje. Sin embargo, según Didham, et al. (1998), no todas las especies se ven igualmente afectadas; este principio, aunque

\footnotetext{
*Correspondencia:

Leonardo A. Ospina-López, ospinaleonardo@gmail.com

Recibido: 5 de abril de 2015

Aceptado: 24 de septiembre de 2015
} 
establecido de manera intuitiva, es fundamental para la forma de abordar los estudios de biodiversidad. Los modelos espaciales de la dinámica de poblaciones dejan claro que la subdivisión del hábitat puede alterar la estabilidad de las poblaciones y las interacciones entre especies en forma contrastante, razón por la cual el estudio de los componentes del paisaje adquiere importancia, ya que su estructura puede determinar los patrones de distribución de las especies. No obstante, uno de los principales problemas con respecto a la capacidad de predicción de los estudios sobre fragmentación, ha sido su enfoque centrado en los fragmentos de bosque y la falta de atención en la matriz adyacente, entendida esta como los hábitats contiguos o alternos al bosque (matorrales y pastizales) (Brosi, et al., 2008).

El departamento de Tolima no ha sido ajeno a estos procesos de transformación de hábitats naturales, pues su reducción a causa de las actividades antropogénicas es evidente. De acuerdo con Vargas, et al. (2007), en el Tolima la modificación de las coberturas boscosas debida al cambio en el uso del suelo ha llevado a procesos de fragmentación con pérdida de conectividad en el paisaje, lo que ha reducido el área de bosque a 295.906 hectáreas. Sin embargo, se presume una alta biodiversidad, situación que ha motivado a instituciones e investigadores a realizar estudios para conocer la diversidad faunística regional en diferentes zonas del departamento (Villa, et al., 2004, 2005a, 2005b, Reinoso, et al., 2007, 2008a, 2008b), lo que ha permitido ampliar el conocimiento sobre algunos grupos como el de los macroinvertebrados acuáticos, las mariposas, los peces, los anfibios, los reptiles, las aves y los mamíferos.

Es claro que aunque en los últimos años se han hecho diversos inventarios y estudios de las mariposas en Colombia, su diversidad no se conoce del todo, sobre todo en algunas áreas geográficas donde hace falta hacer muestreos y considerar que hay grupos en los que se siguen descubriendo especies (Lamas, 2003). Existe, sin embargo, el reporte de 354 especies en el flanco oriental de la cordillera Central colombiana (Andrade-C, 2002). La necesidad de ampliar el conocimiento sobre este grupo de insectos plantea la pregunta sobre cuáles serían las diferencias estructurales del paisaje que determinan los patrones de riqueza y diversidad y cómo estas varían con relación a los diferentes tipos de hábitat, ya que como indicadores biológicos que son, las mariposas se definen como el grupo taxonómico que puede reflejar el estado de la biota en cuanto a la diversidad, a la relación con otras áreas geográficas, a la variación a lo largo de gradientes, a los endemismos o al grado de intervención humana (Fagua, 2001); en general, las mariposas son organismos muy sensibles a los cambios de temperatura, humedad y radiación solar que se producen por la perturbación de su hábitat, por lo cual el inventario de sus comunidades mediante la medición de la diversidad y la riqueza representa una herramienta válida para evaluar el estado de conservación o alteración del medio natural (Kremen, et al., 1993).

\section{Materiales y métodos}

\section{Área de estudio}

La cuenca del río Lagunillas se encuentra en el flanco oriental de la cordillera Central, al norte del departamento del Tolima, y tiene un área de 83.335,51 hectáreas distribuidas en los municipios de Casabianca, Palocabildo, Armero Guayabal, Ambalema, Lérida, Líbano y Villahermosa (Figura 1). El régimen climatológico es bimodal y su temperatura varía entre los $12{ }^{\circ} \mathrm{C}$ en las zonas altas y los $30^{\circ} \mathrm{C}$ en las zonas bajas. Se ubicaron cinco localidades de muestreo distribuidas en el gradiente altitudinal comprendido entre los 345 y 2.950 m (Tabla 1$)$.

\section{Trabajo de campo}

La recolección de las mariposas se hizo mediante dos metodologías: con trampas Van Someren-Rydon y con red entomológica (jama), de acuerdo con las especificaciones de DeVries (1987) y Andrade-C, et al. (2013). Para cada muestreo y en cada localidad de estudio se ubicaron en el interior del bosque durante tres días 12 trampas dispuestas en un transecto de $250 \mathrm{~m}$ de la siguiente manera: seis entre 0 y $1 \mathrm{~m}$ de altura sobre el suelo y seis a más de $5 \mathrm{~m}$ sobre el suelo y a una distancia de $50 \mathrm{~m}$ cada una en el gradiente horizontal. En cuanto a la recolecta con red entomológica, se cubrieron como mínimo cuatro transectos de longitud definida $(250 \mathrm{~m})$ por día de campo en cada hábitat (bosque BS, matorral/rastrojo MA/RA, pastos PA) de cada localidad. La recolección la hacía una sola persona desde las 07:00 hasta 19:00 horas hasta completar 36 horas/jama/hombre y 72 horas/trampa/muestreo en cada sitio visitado, o el equivalente a 12 horas/jama/hombre/hábitat y 72 horas/ trampa/bosque en cada localidad.

Los ejemplares recolectados se sacrificaron por presión digital en el tórax y se almacenaron en sobres triangulares de papel milano blanco con los datos básicos de la recolección (localidad, tipo de hábitat, fecha, altitud, número de recolección, número de transecto, tipo de vegetación predominante, hora, condición climática) (Andrade-C, et al., 2013).

La información de la localización geográfica de cada sitio de muestreo se consignó mediante coordenadas medidas con el programa GPSmap 60 CSx GARMIN a partir de un punto al azar y bordeando cada hábitat o unidad del paisaje en un radio de $0,6 \mathrm{~km}$, con lo que se obtuvo una parcela circular de 113,09 hectáreas.

\section{Métodos de laboratorio}

Para la determinación de algunos ejemplares fue necesaria la disección de su genitalia, para lo cual se siguió el procedimiento propuesto por Birket (1959) con algunas modificaciones personales: se hizo el corte de los cuatro o cinco últimos segmentos abdominales del ejemplar en seco, se los sumergió en una solución líquida de hidróxido 


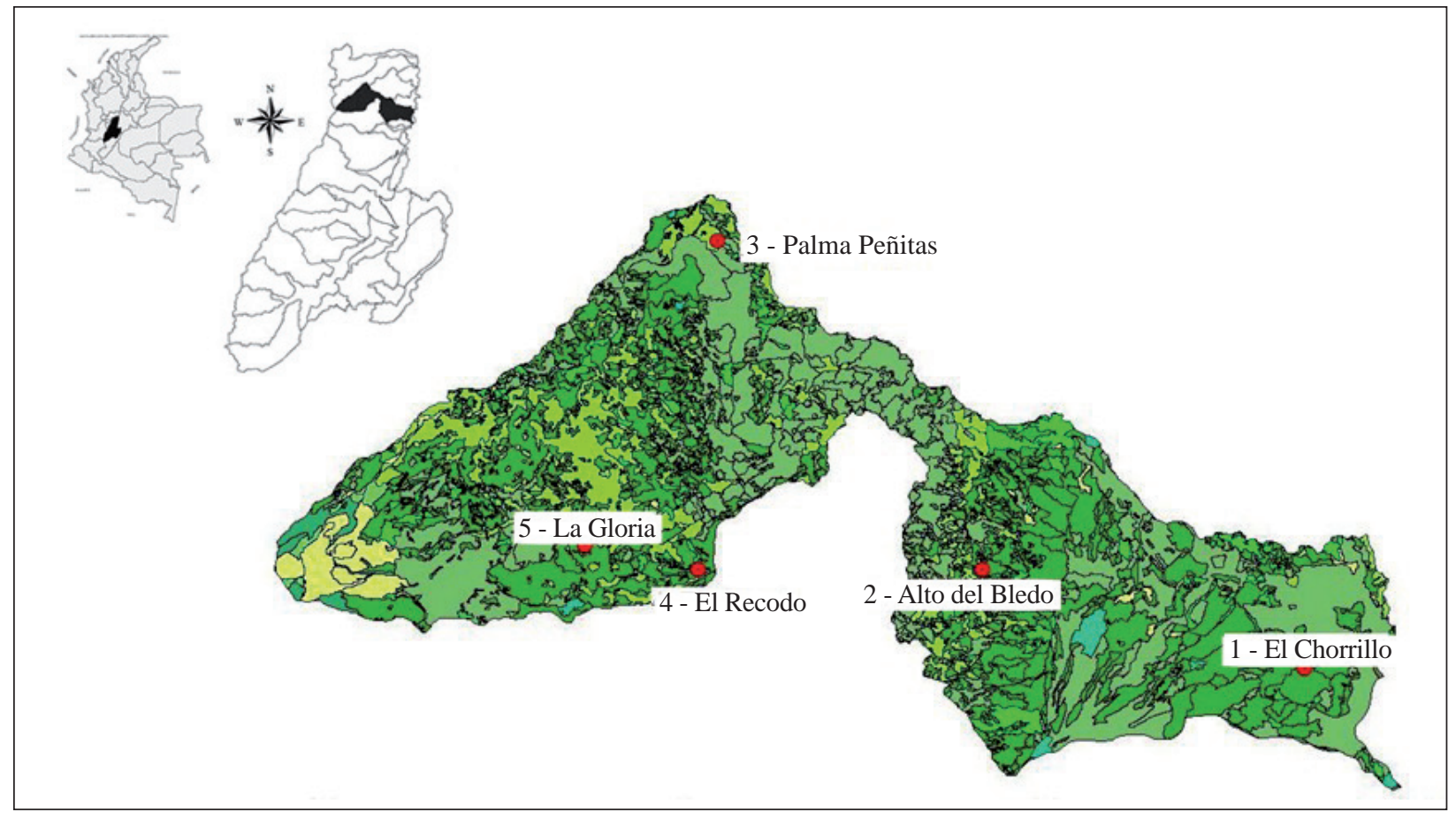

Figura 1. Localización de la cuenca del río Lagunillas, área de estudio y estaciones de muestreo (puntos rojos)

Tabla 1. Estaciones de muestreo seleccionadas en la cuenca del río Lagunillas

\begin{tabular}{|c|c|c|c|c|c|}
\hline No. & Alitud (m) & Municipio & Localidad & Coordenadas & Zonas de vida \\
\hline 1 & 345 & Ambalema & El Chorrillo & $\begin{array}{c}4^{\circ} 50^{\prime} 30.8 ” \mathrm{~N} \\
74^{\circ} 47^{\prime} 23.5 ” O\end{array}$ & Bosque seco tropical (bs-T) \\
\hline 2 & 1.100 & Lérida & Alto del Bledo & $\begin{array}{c}4^{\circ} 53 \text { '40.6”N } \\
74^{\circ} 588^{\prime} 10.8 ” O\end{array}$ & Bosque húmedo premontano (bh-PM) \\
\hline 3 & 1.700 & Casabianca & Palma Peñitas & $\begin{array}{c}5^{\circ} 4^{\prime} 0.9^{\prime \prime} \mathrm{N} \\
75^{\circ} 6.5 .8^{\prime \prime O}\end{array}$ & Bosque húmedo montano bajo (bh-MB) \\
\hline 4 & 2.400 & Líbano & El Recodo & $\begin{array}{l}4^{\circ} 53 ’ 12.3 ” \mathrm{~N} \\
75^{\circ} 6{ }^{\prime} 38.6 ” \mathrm{O}\end{array}$ & Bosque húmedo montano bajo (bh-MB) \\
\hline 5 & 2.950 & Murillo & La Gloria & $\begin{array}{l}4^{\circ} 54^{\prime} 27.6 ” \mathrm{~N} \\
75^{\circ} 10^{\prime} 6.33^{\prime \prime} \mathrm{O}\end{array}$ & Bosque muy húmedo montano (bmh-M) \\
\hline
\end{tabular}

de potasio al $10 \%$ en tubos de ensayo en baño de maría durante 12 o 15 minutos; posteriormente se lavaron con agua destilada y se visualizaron con un estéreomicroscopio para extraer las estructuras esclerotizadas con la ayuda de pinzas, agujas y demás elementos de disección. En cuanto a la determinación taxonómica, se utilizaron las siguientes claves, ilustraciones y descripciones de Seitz (1924), Evans (1951, 1952, 1953, 1955), DeVries (1987), D’Abrera (1981, 1984, 1987a, 1987b, 1988, 1994, 1995), Fox \& Real (1971), Andrade-C (1990, 1995), De La Maza (1987), Álvarez (1993), Constantino (1995), Neild (1996), Tyler, et al. (1994), Le Crom, et al. (2002, 2004), García-Robledo, et al. (2002) y Willmott (2003). Además, se revisó la base de datos (con galería fotográfica) Butterflies of America (Warren, et al., 2013), y cuando no fue posible la determinación de un ejemplar o esta era dudosa, se recurrió a trabajar por comparación con ejemplares de la colección de referencia del Instituto de Ciencias Naturales de la Universidad Nacional de Colombia, sede Bogotá (ICN-MHN-L) y la Colección Zoológica de la Universidad del Tolima, sección Lepidópteros diurnos (CZUT-Ld) (Ibagué-Tolima). Los nombres científicos y la ordenación taxonómica sigue la propuesta de Lamas (2004) y Warren, et al. (2013). Se consignó la información de cada uno de los ejemplares en varias etiquetas: una con los datos de recolección (localidad, recolector, fecha de recolección, coordenadas geográficas y altitud); otra con el número de catálogo de la colección de referencia; una tercera con la información del número del órgano genital extraído para su análisis, y una última con la información taxonómica y el nombre de quien estableció la determinación. 


\section{Análisis de datos}

El análisis de la diversidad biológica se hizo con base en los procedimientos de Magurran (1998, 2004), Moreno (2001) y Lande (1996), y los análisis estadísticos según los métodos planteados por Sokal \& Rohlf (1995). Se usaron los programas Paleontological Statistics, versión 2.15 (Hammer, et al., 2001) y Statistica, versión 7 (StatSoft, 2007). Se describió la riqueza y la diversidad de mariposas a nivel regional de manera general y para los tipos de hábitats. La diversidad se evaluó a partir de la riqueza específica ( $\mathrm{S}=$ número de especies observadas) y el índice de diversidad de Margalef (DMg), en tanto que la abundancia se calculó como el número de individuos correspondientes al registro de una especie por localidad, hábitat y fecha de captura, y a partir de esta se halló la abundancia relativa para cada especie (porcentaje de abundancia). Por otra parte, los índices ecológicos de abundancia proporcional permitieron conocer el grado de uniformidad y el de dominancia de las especies en las localidades y hábitats utilizando los índices de Shannon (H') y de Simpson ( $\lambda$ ).

En cada una de las localidades se estudiaron los elementos del paisaje, clasificados en un sistema de categorías basado en los tipos de ecosistema y diferenciados a partir de características estructurales, las cuales se evidenciaron mediante la exploración de imágenes aéreas disponibles en Google Earth. A partir de estos datos, las imágenes se interpretaron y se digitalizaron mediante un sistema de información geográfica, y con la información tomada en campo se corroboró cada coordenada geográfica para luego digitalizarla y transformarla en una coordenada plana con el programa The Geographic Calculator, versión 3.09 Copyright @ (Blue Marble Geographics, 1992-94). Enseguida, estos datos se analizaron en el programa ArcView ${ }^{\circledR}$, versión 3.2a (Rempel, et al., 2012), con el cual se visualizaron y se unieron punto a punto formando polígonos que corroboraron espacialmente la presencia de los diferentes tipos de coberturas y usos del suelo (unidades paisajísticas). Posteriormente se utilizó la extensión Patch Analyst@ $@$ del programa ArcGIS 9.3 para el análisis del paisaje y la obtención de las características de la métrica en cada localidad y tipo de hábitat.

Caracterización del contexto paisajístico y de los tipos de hábitats. A partir de las parcelas circulares de 0,6 km de radio, se hicieron las diferentes mediciones de las unidades paisajísticas (hábitats). Cada unidad de paisaje se clasificó de la siguiente manera: en el hábitat de bosque (BS), bosque secundario, bosque plantado, bosque natural o bosque alternado con café, caña panelera, plátano, frutales, pasto con rastrojo o enmalezado, pasto manejado y guadua, siempre que la matriz boscosa fuera mayor en área y los componentes alternos se encontraran inmersos en dicha matriz general; en el hábitat de pastos (PA), pasto natural, pasto manejado, pasto con rastrojo o enmalezado, o pastos con pequeños parches de cultivo, tierras eriales, afloramientos rocosos, entre otros, siempre que la matriz dominante fueran pasturas extensas, y en el hábitat de matorrales o rastrojos (MA/ $\mathrm{RA}$ ), rastrojo o rastrojos con pastos naturales o manejados y tierras eriales, siempre que la matriz dominante fueran rastrojos o matorrales.

Medición del contexto paisajístico. Con el programa ArcGIS 9.3 y su extensión Patch Analyst 5.0, se midió el área y el perímetro de cada unidad paisajística por zona de muestreo, lo que permitió hallar los diferentes índices y demás mediciones de la estructura del paisaje (métrica de paisaje) y de los hábitats presentes (métrica a nivel de clase) (McGarigal \& Marks, 1994) (Tabla 2). Además se hizo un análisis de correlación de Spearman (rS) entre las variables de estructura del paisaje y de los hábitats y la abundancia, la riqueza y la diversidad de mariposas.

\section{Resultados}

\section{Composición general}

Se recolectaron 904 ejemplares de mariposas pertenecientes a 266 especies, 115 subespecies, 176 géneros, 20 subfamilias y seis familias (Papilionidae, Pieridae, Lycaenidae, Riodinidae, Nymphalidae y Hesperiidae). En términos generales, la mayor representatividad en cuanto al número de individuos y especies se encontró en el hábitat de matorral o rastrojo (MA/RA) (170 especies, 386 individuos), con $63 \%$ de las especies y $44 \%$ de los individuos, seguido de los hábitats de bosque (BS) (158 especies, 405 individuos) y pastos (PA) (58 especies, 113 individuos). Definida la representatividad mediante el índice de riqueza de Margalef $\left(\mathrm{D}_{\mathrm{Mg}}\right)$, se observó que el hábitat MA/RA tuvo el mayor índice $\left(\mathrm{D}_{\mathrm{Mg}}=28,38\right)$, seguido por el de $\mathrm{BS}\left(\mathrm{D}_{\mathrm{Mg}}=26,15\right)$ y el de PA $\left(\mathrm{D}_{\mathrm{Mg}}=12,06\right)$ (Figura 2).

Tabla 2. Índices y variables para la caracterización del contexto paisajístico y de los tipos de hábitats

\begin{tabular}{ll}
\hline Métrica & Variable \\
\hline Área & Área de clase \\
\hline Densidad de parche & Número de parches \\
\hline Tamaño & Media del tamaño de los parches \\
\hline Variabilidad & $\begin{array}{l}\text { Coeficiente de variación del tamaño } \\
\text { de los parches }\end{array}$ \\
\hline Borde & Borde total \\
\hline Forma & $\begin{array}{l}\text { Índice de la media de la forma } \\
\text { Índice de la forma de la media ponderada } \\
\text { de área }\end{array}$ \\
& Media de la dimensión fractal del parche \\
& $\begin{array}{l}\text { Dimensión fractal de la media ponderada } \\
\text { del área del parche }\end{array}$ \\
\hline Diversidad & $\begin{array}{l}\text { Índice de diversidad de Shannon } \\
\text { Índice de uniformidad de Shannon }\end{array}$ \\
\hline
\end{tabular}


Además, se registraron valores elevados en el índice de equidad de Shannon-Wiener para los hábitats de MA/RA $\left(H^{\prime}=4,76\right)$ y de $B S\left(H^{\prime}=4,67\right)$, a diferencia del hábitat de $\mathrm{PA}$, el cual registró el valor más bajo $\left(H^{\prime}=3,61\right)$; estos valores contrastados mediante una prueba de bondad de ajuste ( $\mathrm{t}$ ) evidenciaron diferencias estadísticamente significativas solo para el hábitat de pastos (BS Vs. MA/RA: $\mathrm{t}=-1,23$, $\mathrm{gl}=787,88, \mathrm{p}=0,21$; BS $V s$. PA: $\mathrm{t}=8,77, \mathrm{gl}=164,08, \mathrm{p}<0,001$; MA/RA Vs. PA: $\mathrm{t}=9,45, \mathrm{gl}=168,3, \mathrm{p}<0,001)$. En cuanto a la dominancia, según el índice de Simpson $(\lambda)$ todos los hábitats presentaron valores bajos $(\lambda<0,1)$, lo que indica que las especies presentes en cada uno de ellos se distribuían uniformemente en relación con su abundancia como se confirmó con los altos valores del índice de Shannon-Wiener. Además, el hábitat de pastos fue más dominante $(\lambda=0,045)$, seguido por el de bosque $(\lambda=0,014)$ y el de matorral o rastrojo $(\lambda=0,013)$ (Figura 3). La prueba de bondad de ajuste mostró diferencias estadísticamente significativas para el hábitat de pastos (BS $V s$. MA/RA: $\mathrm{t}=0,59, \mathrm{gl}=783,96, \mathrm{p}=0,55$; $\mathrm{BS}$ $V s$. PA: $\mathrm{t}=-3,66, \mathrm{gl}=120,08, \mathrm{p}=0,0003$; MA/RA $V s$. PA: $\mathrm{t}=-$ $3,81, \mathrm{gl}=118,56, \mathrm{p}=-0,0002$ ).

Al discriminar por localidades y tipos de hábitats, se encontró que el hábitat de bosque en la localidad Chorrillo (CH) había presentado hasta entonces la mayor riqueza y proporción de individuos (54 especies; $\mathrm{D}_{\mathrm{Mg}}=10,74 ; 139$ individuos), seguida consecutivamente por el de matorrales o rastrojos (34 especies; $\mathrm{D}_{\mathrm{Mg}}=8,23 ; 55$ individuos) y el de pastos (13 especies; $\mathrm{D}_{\mathrm{Mg}}=4,43 ; 15$ individuos), en tanto que en las demás localidades el hábitat de matorrales o rastrojos presentó la mayor riqueza y abundancia, exceptuando la localidad La Gloria (LG) donde este tipo de hábitat no obtuvo registros. El hábitat de pastos en todas las localidades y el de bosque en la localidad Palma Peñitas (PP) registraron los valores más bajos de riqueza y abundancia. Este mismo comportamiento se encontró en los valores de los índices de equidad de Shannon-Wiener ( $\left.\mathrm{H}^{\prime}\right)$ y de dominancia de Simpson ( $\lambda$ ) (Tabla 3). Se encontraron diferencias estadísticamente significativas entre los valores de diversidad (H') de los distintos tipos de hábitats de cada localidad de estudio, excepto entre los del hábitat de bosque y el de matorrales o rastrojos de las localidades Chorrillo, Alto del Bledo y El Recodo y los hábitats de la localidad La Gloria (Tabla 4).

\section{Análisis general del paisaje y su correlación con la diversidad de mariposas}

La descripción general de los hábitats evaluados en la cuenca del río Lagunillas se describe en la Tabla 5, en la que puede verse que el mayor porcentaje de cobertura correspondió al hábitat de pastos en casi todas las localidades de estudio. En la localidad de menor altitud (El Chorrillo, $345 \mathrm{~m}$ ), se encontró que el hábitat predominante era el pasto, seguido por los matorrales o rastrojos y por los bosques; en Alto del Bledo, predominó el pasto seguido por el bosque y los matorrales o rastrojos; en Palma Peñitas, el bosque, seguido

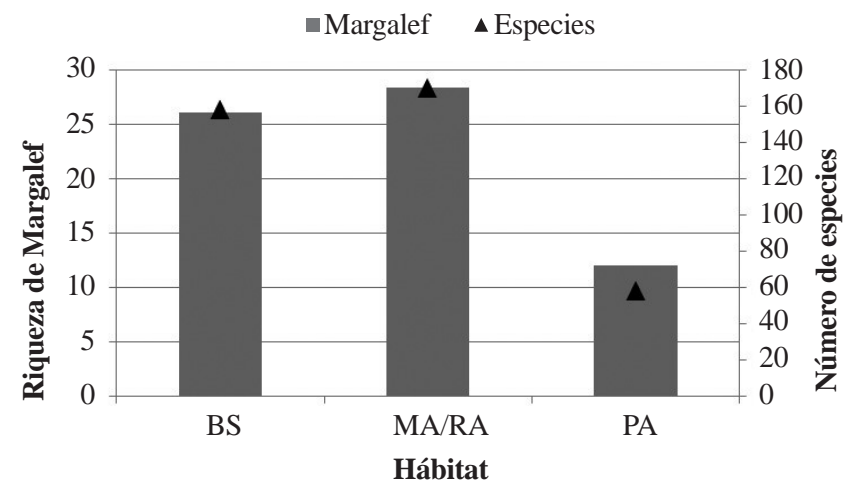

Figura 2. Riqueza específica (número de especies) e índice de Margalef en tres hábitats en la cuenca del río Lagunillas

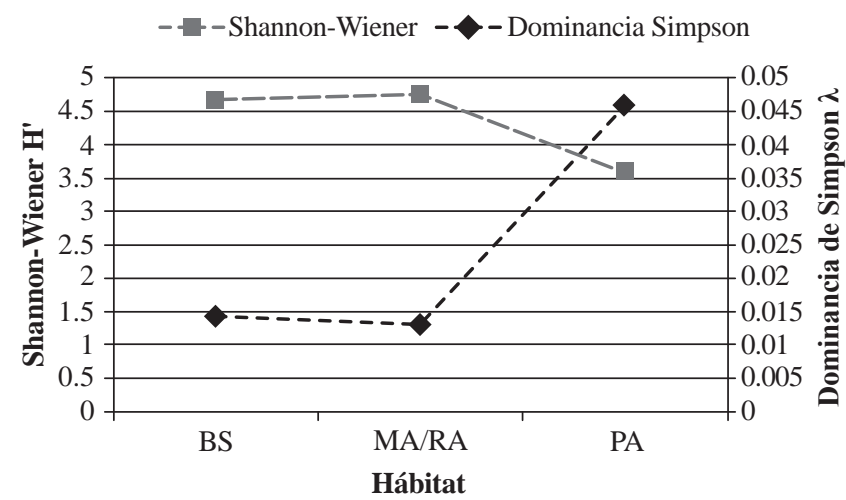

Figura 3. Índices de equidad de Shannon-Wiener (H’) y de dominancia de Simpson $(\lambda)$ para los hábitats encontrados en la cuenca del río Lagunillas

por los matorrales o rastrojos y los pastos; en El Recodo, los pastos seguidos de los matorrales o rastrojos y el bosque, y, por último, La Gloria, los pastos, seguidos de los bosques y los matorrales o rastrojos.

\section{Métrica del paisaje}

El mayor número de parches (NumP) se presentó en la ventana (localidad) de Alto del Bledo (38), seguido por El Chorrillo (27), Palma Peñitas (19), El Recodo (14) y La Gloria (11). Por otra parte, se registró una mayor diversidad en el paisaje de Alto del Bledo (SDI=1,09) y Palma Peñitas $(\mathrm{SDI}=1,08)$, aunque en todas las ventanas los tipos de hábitats se distribuyeron de manera uniforme $(\mathrm{SEI} \geq 0,87)$ (Tabla 6, Figura 4).

Los parches más grandes se presentaron en la ventana La Gloria $(2.950 \mathrm{~m})$, con la mayor media de tamaño de parche (MPS=102.793 $\mathrm{m}^{2}$ ) y el mayor coeficiente de variación $(\mathrm{PSCoV}=230)$. En las demás ventanas el tamaño de los parches y el coeficiente de variación fueron menores, aunque en la ventana Alto del Bledo se evidenció un comportamiento diferente, pues en el paisaje de esta localidad se vieron parches más pequeños 
Tabla 3. Número de especies, abundancia absoluta (individuos), riqueza (índice de Margalef $\mathrm{D}_{\mathrm{Mg}}$ ), diversidad ( $\left.\mathrm{H}^{\prime}\right)$ y dominancia $(\lambda)$ de los hábitats evaluados (BS: bosque, MA/RA: matorral o rastrojo, PA: pastos) en las localidades de estudio: Chorrillo, Alto del Bledo, Palma Peñitas, El Recodo y La Gloria

\begin{tabular}{|c|c|c|c|c|c|c|}
\hline Localidad & Hábitat & Especies & Individuos & $\begin{array}{l}\text { Dominancia Índice } \\
\text { de Simpson } \lambda\end{array}$ & $\begin{array}{l}\text { Índice de Shannon- } \\
\text { Wiener H' }\end{array}$ & $\begin{array}{c}\text { Índice de } \\
\text { Margalef } D_{M g}\end{array}$ \\
\hline \multirow{3}{*}{ Chorrillo } & BS & 54 & 139 & 0,03 & 3,68 & 10,74 \\
\hline & MA/RA & 34 & 55 & 0,04 & 3,37 & 8,24 \\
\hline & $\mathrm{PA}$ & 13 & 15 & 0,08 & 2,52 & 4,43 \\
\hline \multirow{3}{*}{ Alto del Bledo } & BS & 56 & 85 & 0,03 & 3,83 & 12,38 \\
\hline & MA/RA & 72 & 128 & 0,03 & 3,99 & 14,63 \\
\hline & PA & 12 & 14 & 0,09 & 2,44 & 4,17 \\
\hline \multirow{3}{*}{ Palma Peñitas } & BS & 6 & 8 & 0,22 & 1,67 & 2,40 \\
\hline & MA/RA & 59 & 111 & 0,03 & 3,79 & 12,32 \\
\hline & $\mathrm{PA}$ & 20 & 27 & 0,08 & 2,80 & 5,77 \\
\hline \multirow{3}{*}{ EL Recodo } & BS & 38 & 109 & 0,04 & 3,35 & 7,89 \\
\hline & MA/RA & 47 & 92 & 0,05 & 3,47 & 10,17 \\
\hline & $\mathrm{PA}$ & 8 & 16 & 0,15 & 1,98 & 2,53 \\
\hline \multirow[t]{2}{*}{ La Gloria } & BS & 20 & 64 & 0,19 & 2,29 & 4,57 \\
\hline & PA & 12 & 41 & 0,21 & 1,85 & 2,96 \\
\hline
\end{tabular}

Tabla 4. Valores de la prueba de bondad de ajuste (t) para los índices de diversidad de los hábitats en cada localidad de estudio.

\begin{tabular}{|c|c|c|c|c|c|}
\hline \multirow[t]{2}{*}{ Localidad } & \multirow[t]{2}{*}{ Análisis } & \multicolumn{2}{|c|}{ Índice de Shannon-Wiener H' } & \multicolumn{2}{|c|}{ Índice de Simpson $\lambda$} \\
\hline & & $\mathbf{T}$ & $\mathbf{P}$ & $\mathbf{T}$ & $\mathbf{P}$ \\
\hline \multirow{3}{*}{ Chorrillo } & BS $V s . \mathrm{MA} / \mathrm{RA}$ & 2,32 & 0,021 & $-0,89$ & 0,37 \\
\hline & MA/RA $V s$. PA & 3,99 & $<0,001^{*}$ & $-1,34$ & 0,19 \\
\hline & BS $V s$. PA & 5,86 & $<0,001^{*}$ & $-1,66$ & 0,11 \\
\hline \multirow{3}{*}{ Alto del Bledo } & BS $V s . \mathrm{MA} / \mathrm{RA}$ & $-1,22$ & 0,22 & 0,047 & 0,96 \\
\hline & MA/RA $V s$. PA & 7,49 & $<0,001^{*}$ & $-1,84$ & 0,08 \\
\hline & BS $V s$. PA & 6,6 & $<0,001^{*}$ & $-1,83$ & 0,08 \\
\hline \multirow{3}{*}{ Palma Peñitas } & BS $V s . \mathrm{MA} / \mathrm{RA}$ & $-7,37$ & $<0,001^{*}$ & 1,68 & 0,12 \\
\hline & MA/RA $V s$. PA & 4,89 & $<0,001^{*}$ & $-1,64$ & 0,11 \\
\hline & BS $V s$. PA & $-3,44$ & $0,0034^{*}$ & 1,16 & 0,27 \\
\hline \multirow{3}{*}{ El Recodo } & BS $V s$. MA/RA & $-0,87$ & 0,38 & $-0,55$ & 0,57 \\
\hline & MA/RA $V s$. PA & 7,75 & $<0,001^{*}$ & $-2,44$ & $0,025^{*}$ \\
\hline & BS $V s$. PA & 7,86 & $<0,001^{*}$ & $-2,64$ & $0,017^{*}$ \\
\hline La Gloria & BS $V s$. PA & 1,87 & 0,06 & $-0,51$ & 0,605 \\
\hline
\end{tabular}

BS: bosque, MA/RA: matorral o rastrojo, PA: pastos

*diferencias estadísticamente significativas

(MPS $=29.757 \mathrm{~m}^{2}$ ), pero con un coeficiente de variación alto $(\mathrm{PSCoV}=130)$ (Tabla 6, Figura 4). Así, en la mayoría de las ventanas se presentó un número considerable de parches más pequeños con grandes diferencias de tamaño, lo que, sin embargo, se vio afectado por el número de parches.
En relación con la forma de los parches, las métricas indicaron que en todas las ventanas esta fue muy irregular. En La Gloria se presentó el valor más bajo, mientras en las demás ve ntanas la forma promedio general (MSI) y la forma media de los parches (AWMSI) arrojaron valores mayores a 1,84 y 2,74, respectivamente (Tabla 6, Figura 4). 
Tabla 5. Porcentajes de cobertura de los hábitats evaluados generados a partir de una parcela circular de 113,09 hectáreas en cada localidad de muestreo en la cuenca del río Lagunillas

\begin{tabular}{lcccccc}
\hline \multicolumn{7}{c}{ Localidades de muestreo } \\
\hline Tipo de hábitat & Chorrillo & Alto del Bledo & Palma Peñitas & El Recodo & La Gloria & Cuenca (total) \\
\hline Bosque & 23,26 & 29,42 & 31,90 & 18,84 & 21,30 & 24,94 \\
\hline Matorral o rastrojo & 27,76 & 30,63 & 29,10 & 29,68 & 4,40 & 24,31 \\
Pasto & 48,98 & 39,95 & 39,00 & 51,49 & 74,30 & 50,74 \\
\hline
\end{tabular}

Tabla 6. Índices de estructura del paisaje en las cinco localidades bajo estudio y sus respectivos hábitats en la cuenca del río Lagunillas en el departamento del Tolima

\begin{tabular}{|c|c|c|c|c|c|c|c|c|c|c|c|}
\hline Localidad & Hábitat & $\operatorname{AC}\left(\mathbf{m}^{2}\right)$ & NumP & $\operatorname{MPS}\left(\mathrm{m}^{2}\right)$ & PSCoV & TE (m) & MSI & AWMSI & AWMPFD & SDI & SEI \\
\hline \multirow[t]{4}{*}{ Chorrillo } & BS & $262.974,54$ & 4 & $65.743,635$ & 48.815 & $9.587,515$ & 2.658 & 2.786 & 1.408 & - & - \\
\hline & MA/RA & $313.907,23$ & 11 & $28.537,021$ & 133.640 & 14414,006 & 2.248 & 3.051 & 1.426 & - & - \\
\hline & PA & $553.842,95$ & 12 & $46.153,579$ & 72.465 & 20461,209 & 2.241 & 2.551 & 1.383 & - & - \\
\hline & Paisaje (total) & $1.130 .724,73$ & 27 & $41.878,694$ & 89.577 & 44462,729 & 2.306 & 2.745 & 1.401 & 1,05 & 0,96 \\
\hline \multirow{4}{*}{$\begin{array}{l}\text { Alto del } \\
\text { Bledo }\end{array}$} & BS & $332.641,99$ & 9 & $36.960,221$ & 123.902 & 13268,383 & 2.105 & 3.044 & 1.415 & - & - \\
\hline & $\mathrm{MA} / \mathrm{RA}$ & $346.414,61$ & 18 & $19.245,256$ & 82.512 & 17410,852 & 2.074 & 2.161 & 1.398 & - & - \\
\hline & $\mathrm{PA}$ & $451.744,90$ & 11 & $41.067,718$ & 126.992 & 17335,873 & 2.099 & 3.196 & 1.415 & - & - \\
\hline & Paisaje (total) & $1.130 .724,73$ & 38 & $29.757,934$ & 130.372 & 48015,108 & 2.089 & 2.835 & 1.410 & 1,09 & 0,99 \\
\hline \multirow{4}{*}{$\begin{array}{l}\text { Palma } \\
\text { Peñitas }\end{array}$} & BS & 360682,46 & 4 & 90170,615 & 100.500 & 9437,594 & 2.392 & 2.594 & 1.373 & - & - \\
\hline & MA/RA & 329003,73 & 10 & 32900,373 & 93.868 & 13403,589 & 2.194 & 2.299 & 1.386 & - & - \\
\hline & $\mathrm{PA}$ & 441038,54 & 5 & 88207,708 & 82.040 & 15258,256 & 2.984 & 3.361 & 1.422 & - & - \\
\hline & Paisaje (total) & 1130724,73 & 19 & 59511,828 & 111.414 & 38099,439 & 2.444 & 2.807 & 1.396 & 1,08 & 0,98 \\
\hline \multirow[t]{4}{*}{ El Recodo } & BS & $212.987,08$ & 3 & 70.995,693 & 34.056 & 7202,727 & 2.459 & 2.697 & 1.395 & - & - \\
\hline & MA/RA & $335.555,79$ & 6 & $55.925,965$ & 56.097 & 14960,388 & 2.970 & 3.225 & 1.435 & - & - \\
\hline & $\mathrm{PA}$ & $582.181,87$ & 5 & $116.436,375$ & 99.418 & 13475,911 & 2.159 & 2.889 & 1.378 & - & - \\
\hline & Paisaje (total) & $1.130 .724,74$ & 14 & $80.766,053$ & 96.482 & 35639,025 & 2.571 & 2.952 & 1.398 & 1,06 & 0,96 \\
\hline \multirow[t]{4}{*}{ La Gloria } & BS & $240.818,87$ & 9 & $26.757,652$ & 161.487 & 7053,948 & 1.536 & 1.749 & 1.333 & - & - \\
\hline & MA/RA & $49.767,80$ & 1 & $49.767,804$ & 0.000 & 2475,772 & 3.131 & 3.131 & 1.445 & - & - \\
\hline & $\mathrm{PA}$ & 840138,07 & 1 & $840.138,066$ & 0.000 & 10819,533 & 3.330 & 3.330 & 1.362 & - & - \\
\hline & Paisaje (total) & 1130724,74 & 11 & $102.793,158$ & 230.087 & 20349,253 & 1.844 & 2.984 & 1.359 & 0,96 & 0,87 \\
\hline
\end{tabular}

BS: bosque, MA/RA: matorral o rastrojo, PA: pastos. AC: Área de la clase, NumP: Número de parches, MPS: Media del tamaño de los parches, PSCoV: Coeficiente de variación del tamaño de los parches, TE: Borde total, MSI: Índice Media de la forma, AWMSI: Índice de Forma de Media ponderada de área, AWMPFD: Dimensión fractal de la media ponderada del área del parche, SDI: Índice de diversidad de Shannon, SEI: Índice de uniformidad de Shannon.

En términos generales, La Gloria se caracterizó por presentar el paisaje menos heterogéneo, con una menor fragmentación de los hábitats, por cuanto alojaba un menor número de parches y sus formas eran las menos irregulares. En las cinco ventanas restantes se reflejó una mayor heterogeneidad del paisaje debido a que el número de parches (NumP) fue mayor, lo cual se reflejó también en su diversidad (SDI, SEI). Además, la presencia de parches de tamaño promedio mucho menor (MPS), su amplia variabilidad (PSCoV) y la irregularidad de su forma (MSI), aun con mayor peso para los parches más grandes (AWMSI), reflejaron paisajes más fragmentados (Figura 4).

\section{Métrica de los hábitats}

Bosque. A diferencia de los demás hábitats, este tipo tuvo un número menor de fragmentos en toda la cuenca $(\mathrm{NumP}=29)$. En cuanto a su área $(\mathrm{CA}=141 \mathrm{ha})$, presentó un valor intermedio por encima del hábitat de matorrales o rastrojos (132,5 ha) y por debajo del de pastos (286,9 ha). El mayor número de parches se observó en la ventana La Gloria (NumP=9), lo que podría indicar una mayor fragmentación en las zonas con mayor altitud. La mayor extensión de este tipo de hábitat se evidenció en las ventanas Palma Peñitas $(\mathrm{CA}=36,1 \mathrm{ha})$ y Alto del Bledo $(\mathrm{CA}=33,3 \mathrm{ha})$, con mayor número de fragmentos $(\mathrm{NumP}=9)$ y mayor longitud total del 

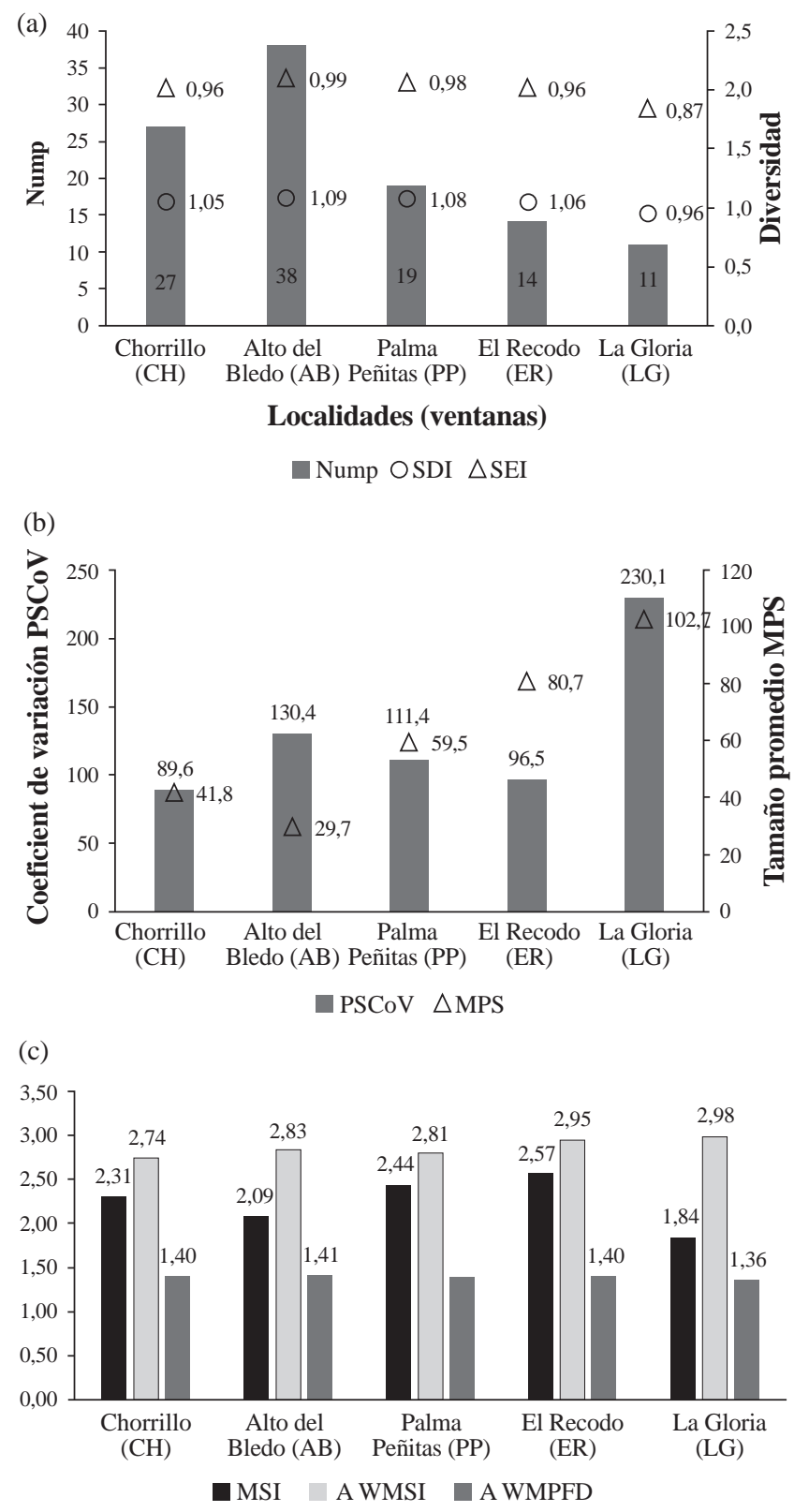

Figura 4. Índices de estructura del paisaje en las localidades de estudio en la cuenca del río Lagunillas

borde $(\mathrm{TE}=13,27 \mathrm{~km})$ en esta última. La menor extensión y número de parches se presentó en la ventana El Recodo $(\mathrm{CA}=21,3$ ha; NumP $=3)$, precedida por la ventana La Gloria, con una extensión de $\mathrm{CA}=24,1$ ha, pero con un número de parches superior (NumP=9) (Figura 5).

En cuanto al tamaño promedio de los parches (MPS), los bosques de las zonas entre los 1.700 y los 2.400 metros de altitud (ventanas Palma Peñitas y El Recodo) presentaron los mayores valores (7,10 Ha y 9,02 ha, respectivamente), seguido por las ventanas El Chorrillo (345 m, 6,57 ha), Alto del Bledo (3,70 ha) y La Gloria (2,68 ha). Por lo tanto, aunque existan bosques con un área considerablemente grande, como es el caso de Alto del Bledo, la longitud de su borde (TE) reflejó la irregularidad de su forma (AWMSI > 3.0), y en bosques como el de La Gloria, con área y longitud de borde menores, se observó una menor irregularidad de forma (AWMSI <1.8) (Figura 5).

Matorrales o rastrojos. Su representatividad fue grande en toda la cuenca en cuanto al número de parches presentes (NumP $=45$ ), pero de menor extensión que en los demás hábitats evaluados $(\mathrm{CA}=132,5 \mathrm{ha})$. El mayor número de parches se registró en la ventana Alto del Bledo ( $\mathrm{NumP}=18)$, seguida por El Chorrillo (11), Palma Peñitas (10), El Recodo (6) y La Gloria (1), esta última con la menor extensión $(\mathrm{CA}=4,97 \mathrm{ha})$ (Figura 5).

Aunque se presentaron valores similares de tamaño o área $(\mathrm{CA}=31,4-34,6)$ y de longitud del borde en todas las ventanas $(\mathrm{TE}=13,4 \mathrm{~m}-14,96 \mathrm{~m})$ con excepción de $\mathrm{La}$ Gloria, cuyos valores fueron inferiores, se evidenciaron formas más irregulares en las ventanas con mayor altitud: El Recodo (2.400 m) (AWMSI=3,22 y AWMPFD=1,43) y La Gloria (2.950 m) (AWMSI=3,13 y AWMPFD=1,44), así como en El Chorrillo (AWMSI=3,05 y AWMPFD=1,42), cuyo coeficiente de variación en el tamaño de los parches fue el más alto (PSCov=133,6). En su conjunto, estos valores reflejan una alta fragmentación de este hábitat en estas zonas (Figura 5).

Pastos. Este hábitat presentó el área más representativa en todas las ventanas $(\mathrm{CA}=286,9 \mathrm{ha})$, pero con un valor intermedio en el número de parches $(\mathrm{NumP}=34)$ comparado con el de los demás hábitats evaluados, valor que disminuyó a medida que aumentaba el gradiente altitudinal, siendo El Chorrillo la ventana con el mayor número de parches (NumP=12), seguida por Alto del Bledo (11), Palma Peñitas (5), El Recodo (5) y La Gloria (1). El área varió entre 44,10 hectáreas en Palma Peñitas y 84,01 en La Gloria. El tamaño promedio de los parches (MPS) aumentó con el gradiente altitudinal, siendo más pequeños los de tierras bajas (El Chorrillo: $\mathrm{AC}=4,61$ ha; Alto del Bledo: $\mathrm{AC}=4,10$ ha) $\mathrm{y}$ más grandes los de tierras altas (El Recodo: $\mathrm{AC}=11,64$ ha; La Gloria: $\mathrm{AC}=84,01$ ha); sin embargo, el coeficiente de variación indicó que la diferencia entre el tamaño de estos parches al interior de cada ventana era grande. Además, la longitud del borde en relación con el área reflejó formas irregulares para este tipo de hábitat (MSI, AWMSI y AWMPFD) (Figura 5).

A pesar de que el hábitat de pastos fue el menos fragmentado (es decir, poco subdividido) y el más representativo en términos de área, con poca irregularidad en su forma, ello puede ser el reflejo de un bajo grado de transformación. En contraposición, el hábitat de matorrales o rastrojos fue el de menor extensión en el paisaje y el más regular en cuanto a la forma, aunque el mayor número de parches en él podría indicar una mayor fragmentación de este hábitat. 
(a)

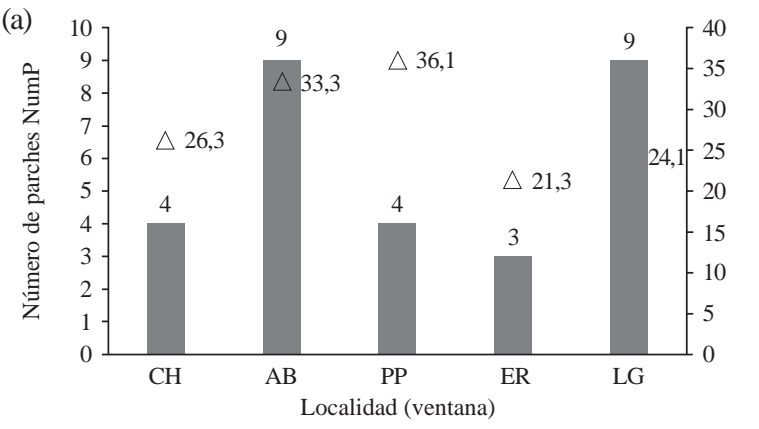

- NumP $\triangle \mathrm{CA}$

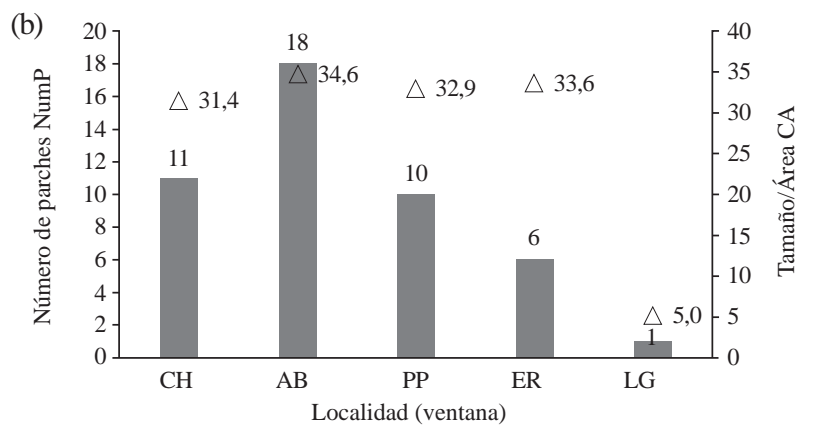

numP $\triangle \mathrm{CA}$

(c)

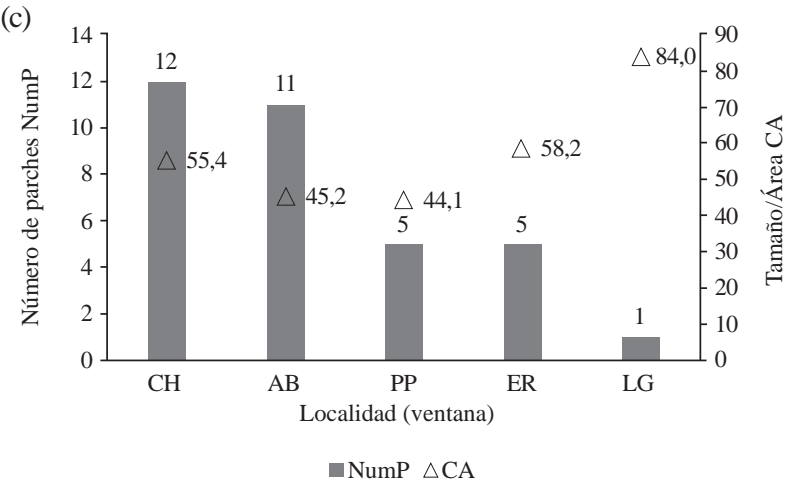

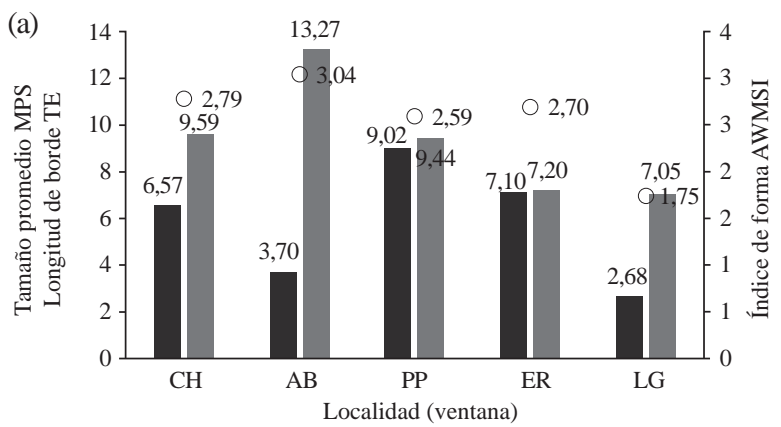

- MPS —TE OAWMSI

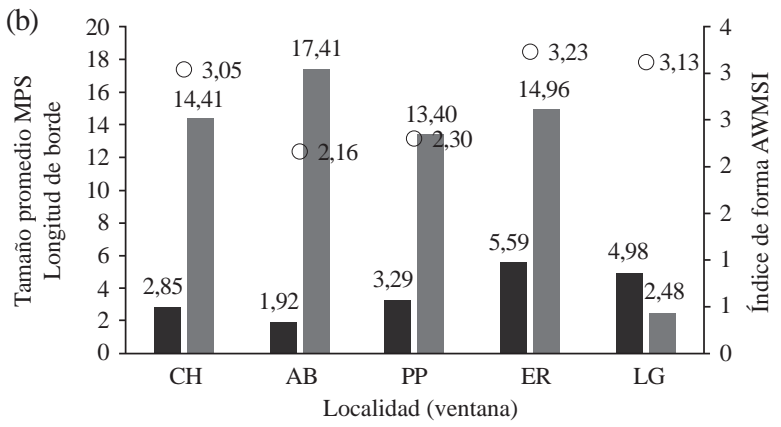

-MPS $\square$ TE OAWMSI

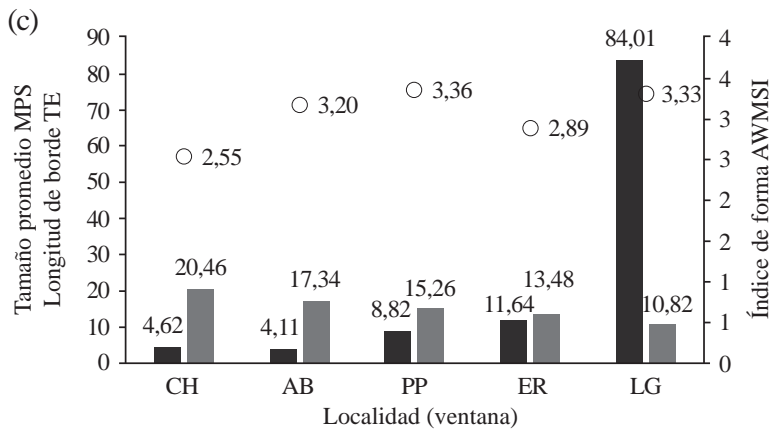

- MPS -TE OAWMSI

Figura 5. Índices de estructura para los tres tipos de hábitats en las localidades de estudio en la cuenca del río Lagunillas: (a) bosque (BS), (b) matorral o rastrojo (MA/RA) y (c) pasto (PA)

\section{Paisajes y hábitats y su relación con las mariposas}

Paisaje. Se hallaron algunas correlaciones entre el número de especies y de individuos, la riqueza, la diversidad y la dominancia de mariposas y los índices de métrica del paisaje. El número de especies y de individuos registrados se correlacionaron positivamente $(r S=0,9 ; \mathrm{p}=0,037)$ con la dimensión fractal dela media ponderada delárea(AWMPFD), calculada esta como el grado de complejidad del paisaje a partir de la relación entre el área y el perímetro de todos los parches evaluados; en términos generales esto implica que a medida que el paisaje se hace más irregular en su forma, la cantidad de individuos y de especies aumenta. La diversidad (H') de mariposas en toda la cuenca se correlacionó positivamente con el número de parches presentes en el paisaje (NumP) y la longitud del borde de los fragmentos
(TE) $(r S=0,9 ; \mathrm{p}=0,037)$, pero negativamente con el tamaño promedio de los parches (MPS) $(r S=-0,9 ; \mathrm{p}=0,037)$, es decir, que a mayor número de fragmentos pequeños con formas mucho más irregulares en el paisaje, mayor fue la diversidad de las especies de mariposas. Este comportamiento se reconfirmó con las correlaciones encontradas entre el índice de dominancia de Simpson $(\lambda)$ y las mismas variables de paisaje, pero de una manera recíproca. Además, la mayor diversidad espacial del paisaje (SDI, SEI) podría ser la causa de la mayor riqueza de mariposas $(r S=0,9 ; \mathrm{p}=0,037)$ (Figura 6, Tabla 7).

Hábitats. En el caso del hábitat de bosque solo se encontraron correlaciones positivas en cuanto al número de especies, la riqueza $\left(\mathrm{D}_{\mathrm{Mg}}\right)$ y la diversidad (H') de mariposas, y una correlación negativa de la dominancia frente a los índices 

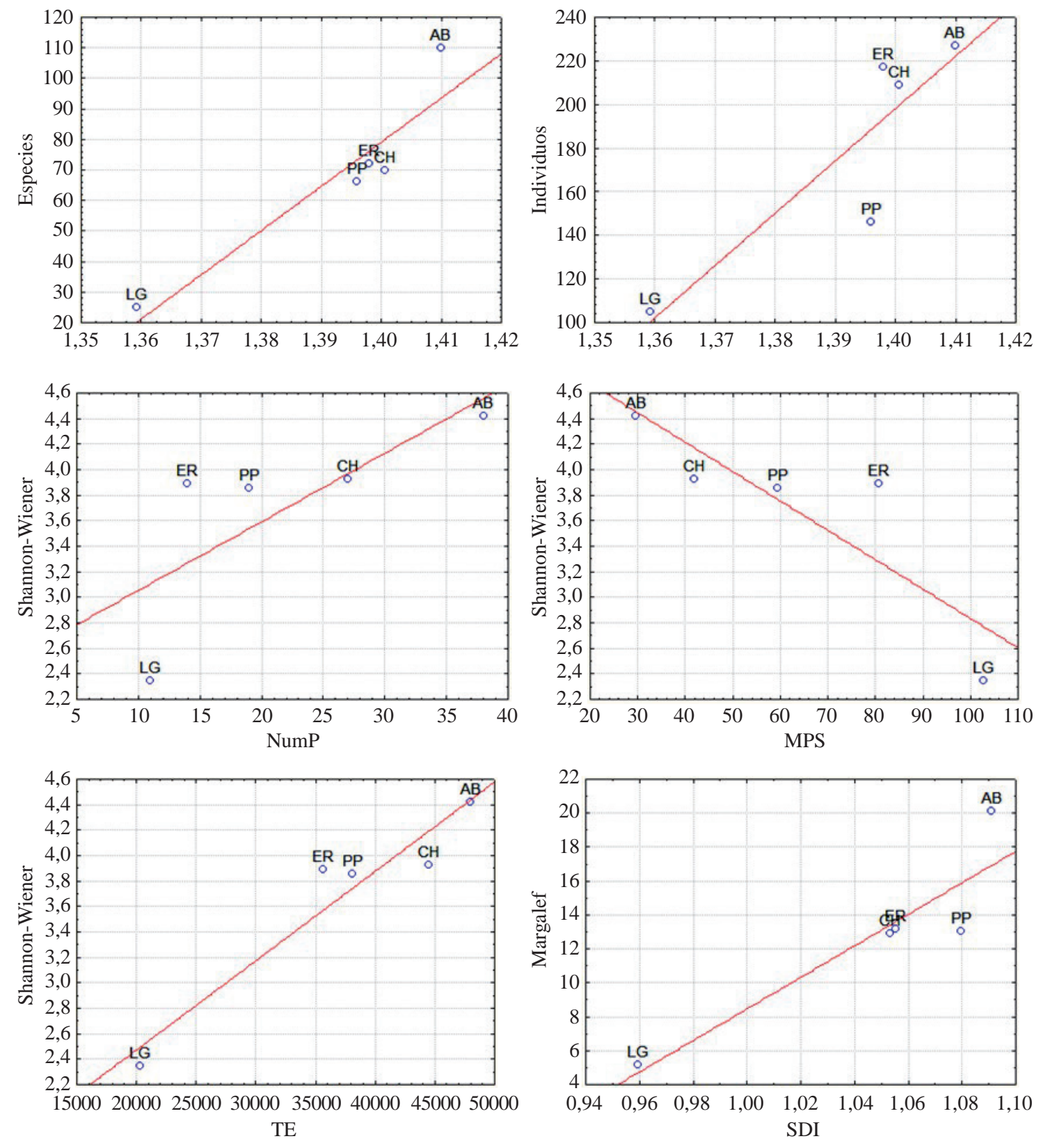

Figura 6. Correlaciones significativas entre variables de paisaje y valores de riqueza y diversidad para las mariposas de la cuenca del río Lagunillas

de forma (AWMSI y AWMPFD) de los bosques $(r S=0,9$; $\mathrm{p}=0,037 ; r S=-0,9 ; \mathrm{p}=0,037$, respectivamente) (Figura 6, Tabla 7. Cuanto más irregular (AWMSI) y compleja era la forma (AWMPFD) de los fragmentos de bosque en el paisaje, mayor el número de especies y, por ende, mayor la riqueza y la diversidad de mariposas (Tabla 7, Figura 7). En cuanto al hábitat de matorrales o rastrojos, aunque los valores del coeficiente de Spearman (rS) fueron altos, no se encontraron correlaciones significativas $(\mathrm{rS}=0,8$; $\mathrm{p}>0,05$ ) entre los distintos valores de riqueza y diversidad de mariposas y las características estructurales del paisaje en las ventanas (Tabla 7).
Por el contrario, en el hábitat de pastos se registraron correlaciones significativas entre el número de individuos, la diversidad y la dominancia y las métricas de tamaño (CA), variabilidad y forma (MSI, AWMPFD) de los parches (Tabla 7). La extensión (AC) de los pastos se correlacionó de manera negativa con la diversidad $(r S=-0,9 ; \mathrm{p}=0,037)$ y positivamente con la dominancia $(r S=0,9 ; \mathrm{p}=0,037)$; el tamaño promedio de los parches (MPS) y su variabilidad de formas (MSI) explican este fenómeno, y su correlación con el número de individuos fue positiva $(r S=0,9 ; \mathrm{p}=0,037)$, lo que implica que la presencia de parches de gran tamaño pero muy variables en su forma permitiría explicar la gran 
Tabla 7. Resultados del coeficiente de correlación de Spearman $(r S)$ para el número de especies, número de individuos, riqueza de mariposas según el índice de Margalef $\left(\mathrm{D}_{\mathrm{Mg}}\right)$, diversidad según el índice de Shannon-Wiener $\left(\mathrm{H}^{\prime}\right)$ y dominancia según el índice de Simpson $(\boldsymbol{\lambda})$ en relación con la métrica a nivel de paisaje y los tipos de hábitat.

\begin{tabular}{|c|c|c|c|c|c|c|c|c|c|c|}
\hline \multirow[t]{2}{*}{ Clases / Análisis } & \multicolumn{2}{|c|}{ No. de especies } & \multicolumn{2}{|c|}{ No. de individuos } & \multicolumn{2}{|c|}{ Riqueza (DMg) } & \multicolumn{2}{|c|}{ Diversidad (H') } & \multicolumn{2}{|c|}{ Dominancia $(\lambda)$} \\
\hline & $r S$ & $\mathrm{P}$ & $r s$ & $\mathrm{p}$ & $r S$ & $\mathrm{p}$ & $r S$ & $\mathrm{p}$ & $r S$ & $\mathrm{p}$ \\
\hline \multicolumn{11}{|l|}{ Paisaje } \\
\hline NumP & 0,70 & 0,188 & 0,70 & 0,188 & 0,60 & 0,285 & 0,90 & $0,037^{*}$ & $-0,90$ & $0,037 *$ \\
\hline MPS & $-0,70$ & 0,188 & $-0,70$ & 0,188 & $-0,60$ & 0,285 & $-0,90$ & $0,037^{*}$ & 0,90 & $0,037 *$ \\
\hline PSCoV & $-0,30$ & 0,624 & $-0,30$ & 0,624 & $-0,10$ & 0,873 & $-0,40$ & 0,505 & 0,40 & 0,505 \\
\hline $\mathrm{TE}$ & 0,70 & 0,188 & 0,70 & 0,188 & 0,60 & 0,285 & 0,90 & $0,037^{*}$ & $-0,90$ & $0,037 *$ \\
\hline MSI & 0,30 & 0,624 & 0,30 & 0,624 & 0,40 & 0,505 & 0,10 & 0,873 & $-0,10$ & 0,873 \\
\hline AWMSI & $-0,20$ & 0,747 & $-0,20$ & 0,747 & $-0,10$ & 0,873 & $-0,50$ & 0,391 & 0,50 & 0,391 \\
\hline AWMPFD & 0,90 & $0,037^{*}$ & 0,90 & $0,037^{*}$ & 0,70 & 0,188 & 1,00 & - & $-1,00$ & - \\
\hline SDI & 0,70 & 0,188 & 0,70 & 0,188 & 0,90 & $0,037^{*}$ & 0,60 & 0,285 & $-0,60$ & 0,285 \\
\hline SEI & 0,70 & 0,188 & 0,70 & 0,188 & 0,90 & $0,037 *$ & 0,60 & 0,285 & $-0,60$ & 0,285 \\
\hline \multicolumn{11}{|l|}{ Bosque } \\
\hline CA & $-0,10$ & 0,873 & $-0,50$ & 0,391 & $-0,10$ & 0,873 & $-0,10$ & 0,873 & 0,10 & 0,873 \\
\hline NumP & 0,21 & 0,734 & $-0,37$ & 0,541 & 0,21 & 0,734 & 0,21 & 0,734 & $-0,21$ & 0,734 \\
\hline MPS & $-0,40$ & 0,505 & $-0,10$ & 0,873 & $-0,40$ & 0,505 & $-0,40$ & 0,505 & 0,40 & 0,505 \\
\hline PSCoV & $-0,10$ & 0,873 & $-0,60$ & 0,285 & $-0,10$ & 0,873 & $-0,10$ & 0,873 & 0,10 & 0,873 \\
\hline $\mathrm{TE}$ & 0,70 & 0,188 & 0,30 & 0,624 & 0,70 & 0,188 & 0,70 & 0,188 & $-0,70$ & 0,188 \\
\hline MSI & 0,20 & 0,747 & 0,70 & 0,188 & 0,20 & 0,747 & 0,20 & 0,747 & $-0,20$ & 0,747 \\
\hline AWMSI & 0,90 & $0,037^{*}$ & 0,60 & 0,285 & 0,90 & $0,037 *$ & 0,90 & $0,037^{*}$ & $-0,90$ & $0,037 *$ \\
\hline AWMPFD & 0,90 & $0,037^{*}$ & 0,60 & 0,285 & 0,90 & $0,037 *$ & 0,90 & $0,037^{*}$ & $-0,90$ & $0,037 *$ \\
\hline \multicolumn{11}{|c|}{ Matorral/Rastrojo } \\
\hline CA & 0,80 & 0,200 & 0,80 & 0,200 & 0,80 & 0,200 & 0,80 & 0,200 & $-0,40$ & 0,600 \\
\hline NumP & 0,40 & 0,600 & 0,40 & 0,600 & 0,40 & 0,600 & 0,40 & 0,600 & $-0,80$ & 0,200 \\
\hline MPS & $-0,40$ & 0,600 & $-0,40$ & 0,600 & $-0,40$ & 0,600 & $-0,40$ & 0,600 & 0,80 & 0,200 \\
\hline PSCoV & $-0,40$ & 0,600 & $-0,40$ & 0,600 & $-0,40$ & 0,600 & $-0,40$ & 0,600 & $-0,20$ & 0,800 \\
\hline $\mathrm{TE}$ & 0,40 & 0,600 & 0,40 & 0,600 & 0,40 & 0,600 & 0,40 & 0,600 & $-0,20$ & 0,800 \\
\hline MSI & $-0,80$ & 0,200 & $-0,80$ & 0,200 & $-0,80$ & 0,200 & $-0,80$ & 0,200 & 1,00 & - \\
\hline AWMSI & $-0,80$ & 0,200 & $-0,80$ & 0,200 & $-0,80$ & 0,200 & $-0,80$ & 0,200 & 1,00 & - \\
\hline AWMPFD & $-0,60$ & 0,400 & $-0,60$ & 0,400 & $-0,60$ & 0,400 & $-0,60$ & 0,400 & 0,80 & 0,200 \\
\hline \multicolumn{11}{|l|}{ Pasto } \\
\hline CA & $-0,67$ & 0,219 & 0,40 & 0,505 & $-0,80$ & 0,104 & $-0,90$ & $0,037^{*}$ & 0,90 & $0,037 *$ \\
\hline NumP & 0,26 & 0,669 & $-0,87$ & 0,054 & 0,41 & 0,493 & 0,56 & 0,322 & $-0,56$ & 0,322 \\
\hline MPS & $-0,31$ & 0,614 & 0,90 & $0,037^{*}$ & $-0,50$ & 0,391 & $-0,60$ & 0,285 & 0,60 & 0,285 \\
\hline PSCoV & $-0,31$ & 0,614 & $-0,70$ & 0,188 & $-0,10$ & 0,873 & 0,20 & 0,747 & $-0,20$ & 0,747 \\
\hline TE & 0,46 & 0,434 & $-0,80$ & 0,104 & 0,60 & 0,285 & 0,70 & 0,188 & $-0,70$ & 0,188 \\
\hline MSI & 0,41 & 0,493 & 0,90 & $0,037^{*}$ & 0,20 & 0,747 & $-0,10$ & 0,873 & 0,10 & 0,873 \\
\hline AWMSI & 0,36 & 0,553 & 0,60 & 0,285 & 0,30 & 0,624 & 0,10 & 0,873 & $-0,10$ & 0,873 \\
\hline AWMPFD & 0,67 & 0,219 & $-0,40$ & 0,505 & 0,80 & 0,104 & 0,90 & $0,037^{*}$ & $-0,90$ & $0,037^{*}$ \\
\hline
\end{tabular}

AC: Área de la clase, NumP: Número de parches, MPS: Media del tamaño de los parches, PSCoV: Coeficiente de variación del tamaño de los parches, TE: Borde total, MSI: Índice Media de la forma, AWMSI: Índice de Forma de Media ponderada de área, AWMPFD: Dimensión fractal de la media ponderada del área del parche, SDI: Índice de diversidad de Shannon, SEI: Índice de uniformidad de Shannon, *0,037 correlacion significativa (p<0,05). 

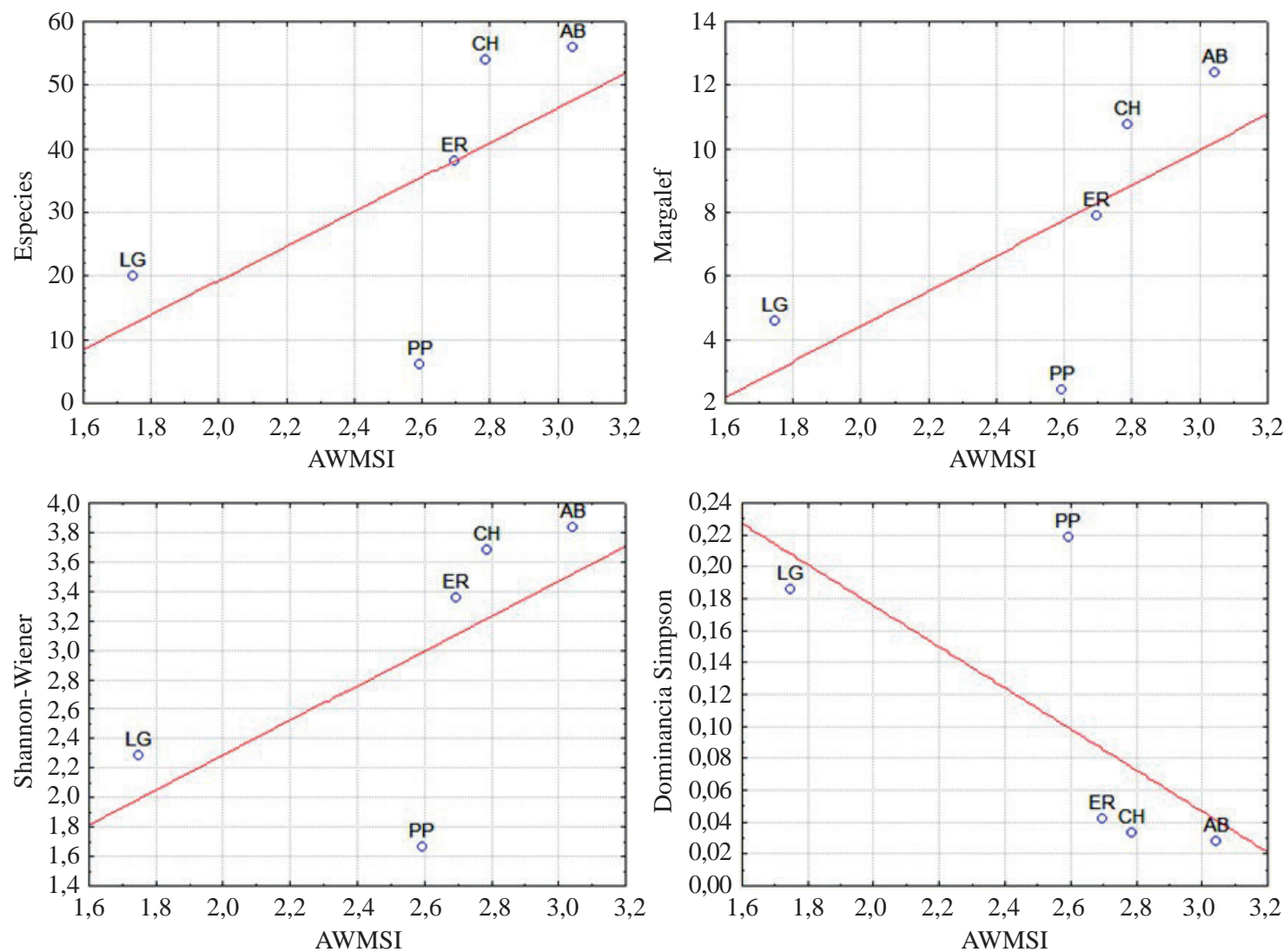

Figura 7. Correlaciones significativas entre variables de paisaje y valores de riqueza y diversidad de mariposas de los bosques de la cuenca del río Lagunillas

abundancia y afectar, por otra parte, la diversidad del grupo. Sin embargo, la correlación positiva de la diversidad y negativa de la dominancia con la forma irregular de los parches (AWMPFD) $(r S=0,9 ; \mathrm{p}=0,037$ y $r S=-0,9 ; \mathrm{p}=0,037$, respectivamente), implicaría que en este hábitat se mantuvo una gran diversidad cuanto más irregulares las formas de sus parches (Figura 8).

\section{Discusión}

La lepidopterofauna encontrada en la cuenca del río Lagunillas en el departamento del Tolima representa más del $8 \%$ de la fauna de lepidópteros reportada para el país, la que según Andrade-C, et al. (2007) es de 3.274 especies, valores que podrían incrementarse cuando se hagan más estudios. De esta manera, es de suponer que áreas pequeñas (vistas desde una escala regional), como es el caso de esta cuenca, podrían albergar una gran riqueza y diversidad de este grupo de insectos, pues se encontraron las seis familias reportadas para el neotrópico y Colombia y 20 subfamilias de las 26 registradas hasta el momento (Warren, et al., 2013).

La mayor riqueza registrada en las localidades de Alto del Bledo (1.100 m) y El Recodo $(2.400 \mathrm{~m})$ puede ser el reflejo de un "dominio intermedio"; en este sentido, Colwell \&
Hurtt (1994) proponen que los picos de riqueza en alturas intermedias son generados por el solapamiento de los rangos altitudinales de las especies de zonas bajas y altas de una región en un punto determinado (dominio); es probable, entonces, que entre estas altitudes se presente la mayor convergencia de especies representantes de zonas bajas y altas, relacionadas con formaciones vegetales subtropicales y montanas. Estos picos de riqueza en alturas intermedias han sido registrados por Fleishman, et al. (1998) en mariposas y por Sanders (2002) en hormigas; otros estudios de mariposas han demostrado este mismo comportamiento en el departamento del Tolima (García-Pérez \& OspinaLópez, 2004; García-Pérez, et al., 2007, Peña-Cerpa, 2007, Ospina-López, et al., 2010).

Por otra parte, a partir de esta cota altitudinal la riqueza comienza a decrecer debido a condiciones ambientales restrictivas como la humedad, la radiación solar, las bajas temperaturas y la neblina, la cual tiende a aumentar la humedad ambiental y a reducir la evapotranspiración, lo que representa una barrera para las especies carentes de respuestas fisiológicas que les permitan establecerse en zonas de alta montaña, además de posibles restricciones alimenticias para los estadios inmaduros. Erelli, et al. (1998) consideran que las bajas temperaturas restringen el 

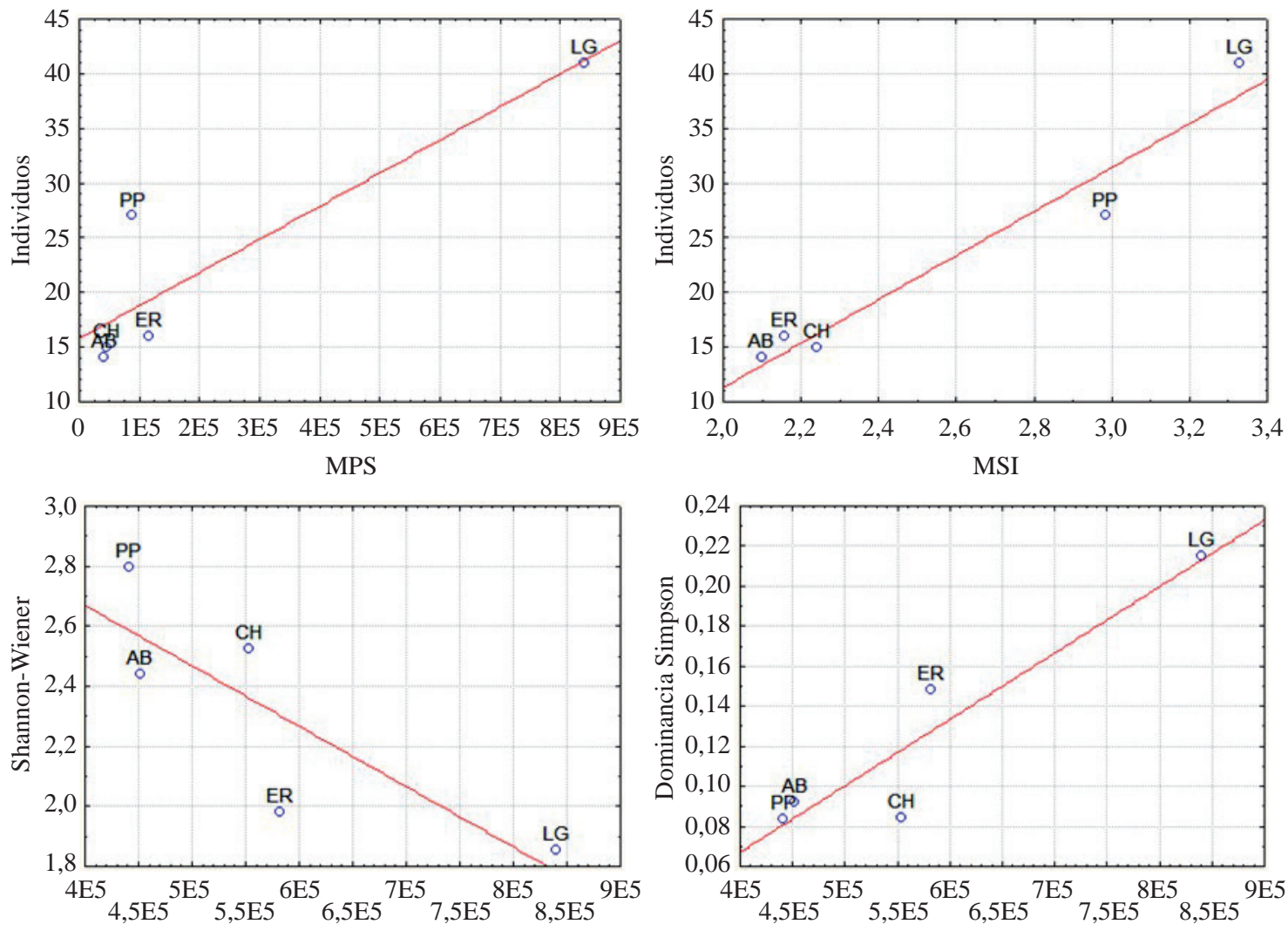

CA
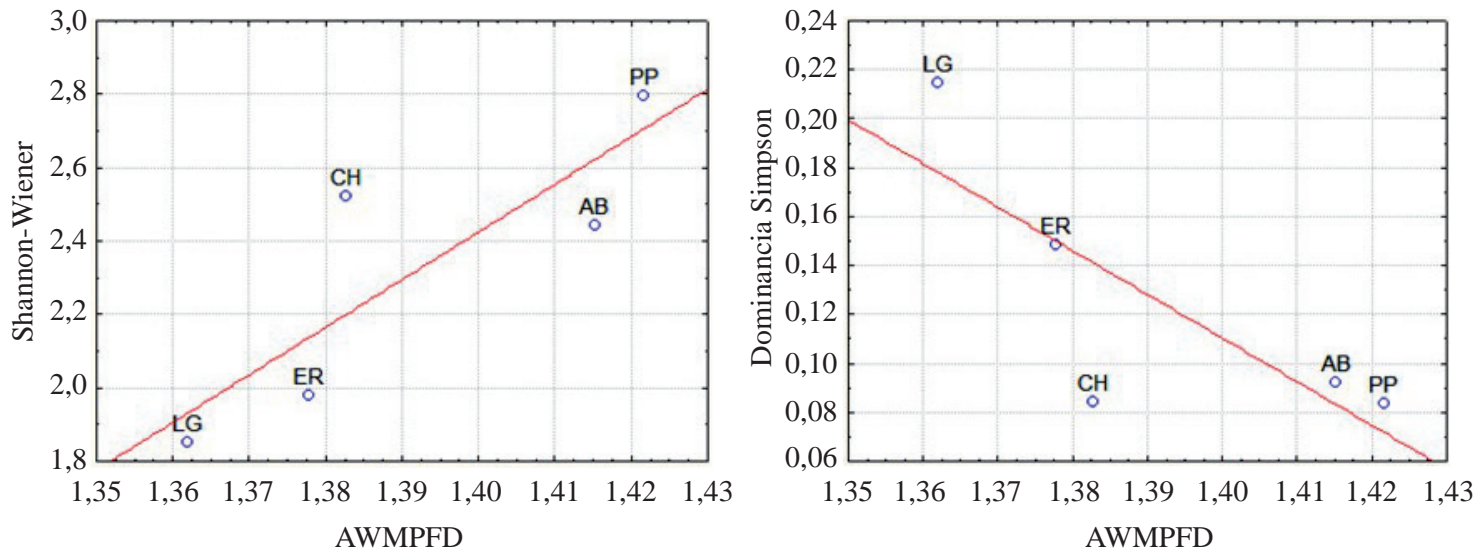

Figura 8. Correlaciones significativas entre variables de paisaje y valores de riqueza y diversidad de mariposas de los pastos de la cuenca del río Lagunillas

crecimiento de las plantas más que la fotosíntesis, lo que conduce a un aumento en los metabolitos secundarios, haciéndolas menos apetecibles para los herbívoros. Este comportamiento de disminución de la riqueza es conocido como regla o efecto de Rapoport (Stevens, 1992, Blackburn \& Gaston, 1996), el cual propone que la riqueza de las especies decrece a medida que la elevación aumenta; en Colombia esta situación ha sido registrado por Bastidas, et al. (2002) en las mariposas del Santuario de Flora y Fauna Galeras (Nariño) y por Fagua (1999) en la cuenca del río Gazunta (Medina-Cundinamarca).
En este estudio se evaluaron varios tipos de hábitats en los cuales se vieron marcadas diferencias en abundancia, riqueza y diversidad. El hábitat de matorrales o rastrojos, considerado como una zona de transición entre dos tipos de hábitat (pastos y bosque), presentó el mayor número de individuos y especies, lo que se asume es el resultado de la interacción entre hábitats adyacentes (Odum, 1971). Esta zona presenta gran variedad de microclimas y condiciones físicas como el régimen de luz, temperatura y humedad, y factores estructurales como la composición vegetal, que influyen en la comunidad de lepidópteros diurnos. Según 
Simonson, et al. (2001), la abundancia y la riqueza de las especies de mariposas puede verse favorecida directamente por las condiciones físicas del hábitat que determinan la tolerancia fisiológica de las especies, la que según Murcia (1995), también puede causar cambios en las interacciones ecológicas como la depredación y la competencia. Estos resultados son similares a los de Hill, et al. (2001) y Schulze, et al. (2004), quienes encontraron que la composición de las mariposas era diferente en las comunidades de bosque natural, pastizales y demás áreas abiertas, pero similar en bosques y en vegetación secundaria (matorrales y rastrojos).

El hábitat de bosque presentó valores similares de riqueza y diversidad, lo que podría indicar que la diversidad de insectos herbívoros, entre ellos las mariposas, se encuentra estrechamente relacionada con la diversidad tanto florística como estructural de la vegetación, ya que los bosques exhiben un incremento en la biomasa vegetal mayor que en los hábitats de borde y de pradera, lo que permite el establecimiento de un mayor número de especies fitófagas (Murdoch, et al., 1972). Además, según Southwood, et al. (1979), la heterogeneidad del hábitat (complejidad estructural de la vegetación) cambia en el curso de la sucesión vegetal, de manera que en los estadios tempranos de sucesión (frecuentes en pastizales), la diversidad de insectos se incrementa con la diversidad de plantas, mientras que en los estadios tardíos (frecuentes en bosques), la diversidad estaría determinada por la heterogeneidad del hábitat. En el caso del bosque, dicha heterogeneidad se define por el número y la distribución de estratos, los cuales determinan condiciones microclimáticas específicas para la comunidad de mariposas. Se puede confirmar, entonces, la importancia de los hábitats boscosos para la conservación de mariposas en paisajes agrícolas, como han demostrado diversos estudios (Ricketts, et al., 2001, Horner-Devine, et al., 2003, Harvey, et al., 2006, Tobar-López, et al., 2007).

La disminución en la riqueza y la diversidad de mariposas en los pastos podría relacionarse con los cambios en la composición y la alteración del crecimiento y la diversidad vegetal ocasionadas por la ganadería intensiva (Krues \& Tschrnke, 2002), lo cual fue evidente en todas las localidades evaluadas. Estos cambios en la vegetación hacen que los parámetros microclimáticos (temperatura, humedad, radiación solar) sean intensos en este hábitat e incidan en la distribución, la composición y la riqueza de las especies de mariposas (Collinge, et al., 2003). Sin embargo, en este estudio fue evidente que, a diferencia de otros sistemas (cultivos), los pastos mantenían una riqueza y abundancia de especies significativas en el paisaje fragmentado, ya que podían conservar una variedad mayor dependiendo de la composición de las especies vegetales y del arreglo espacial del paisaje. Si los pastos se encuentran en cercanías de los fragmentos de bosque o entre ellos permiten una conectividad estructural en el paisaje, y son eficaces en la conservación de la biodiversidad (Harvey, et al., 2000).
En el paisaje de la cuenca del río Lagunillas se observó un porcentaje mayor de pastos, en comparación con los otros hábitats evaluados; sin embargo, en las localidades (ventanas) intermedias, ubicadas entre los $1.000 \mathrm{y}$ los 1.700 m, se observó una mayor proporción de matorrales o rastrojos y de bosques, aunque es importante aclarar que a mayor altitud (ventana La Gloria), el contexto paisajístico presentaba sobre todo pequeños fragmentos de bosque muy afectados por la acción del hombre y de pastos que se extendían como una matriz adyacente, en tanto que el hábitat de matorrales o rastrojos podría considerarse como una matriz fragmentada, aislada y degradada de lo que anteriormente era el bosque (según observaciones de los pobladores de la zona). Este es el resultado de procesos intensivos de fragmentación por causas antrópicas, comunes en la región andina de nuestro país en altitudes entre los 1.000 y los $2.000 \mathrm{~m}$ y en el clima húmedo, donde el paisaje, densamente poblado, se utiliza intensamente para diversas formas de cultivo y queda muy poco del bosque original (Etter \& van Wyngaarden, 2000). Los patrones de transformación en los Andes colombianos, en particular, muestran tendencias históricas diferentes a las de las tierras bajas, ya que su tasa de transformación es mayor (Etter, 1993, Etter \& van Wyngaarden, 2000). Sin embargo, en la cuenca del río Lagunillas el número de parches en el paisaje disminuyó a medida que se ascendía en el gradiente altitudinal, siendo las localidades (ventanas) ubicadas entre los 345 y los $1.100 \mathrm{~m}$ las de mayor número, lo que implicaría que la pérdida de los hábitats naturales ha sido mayor en las zonas intermedias y de mayor altitud, donde los paisajes con parches de mayor tamaño promedio (MPS $>10$ Ha) correspondían a pastizales (pastos) amplios y extensos.

De particular interés resulta la tendencia al aumento en el número de especies e individuos de mariposas a medida que el paisaje se hace más complejo (AWMPDF), complejidad ésta dada por la gran fragmentación del paisaje debida a las actividades antrópicas (McGarigal \& Marks, 1994, McGarigal, et al., 2002). El presente estudio contribuye a demostrar que el factor que limita la abundancia y la riqueza de mariposas depende del contexto de un paisaje local (Ekroos \& Kuussaari, 2012) complejo, heterogéneo e irregular. No obstante, en otro estudio se demostró lo contrario, es decir, que el número de especies e individuos disminuía a medida que la alteración de los hábitats presentes en el paisaje era mayor (Vu, 2009). En este estudio, la riqueza de mariposas en la cuenca aumentó significativamente con el incremento de la diversidad del paisaje (SDI), comportamiento que según Kuussaari, et al. (2009), puede verse en varios grupos taxonómicos, cuyo número aumenta considerablemente con la diversidad del paisaje circundante; además, esas mismas variables del paisaje tienen una fuerte influencia sobre el ensamble de las mariposas, que necesitan de una estructura del paisaje compleja para su desplazamiento y para otras funciones ecológicas, tal y como lo señalan Jeanneret, et al., (2003). 
El número de especies aumenta significativamente a medida que aumenta el tamaño (área) de los hábitats que componen el paisaje, lo que confirma la relación existente entre área y especies (a mayor área, más especies), tal como se ha demostrado en otras comunidades de mariposas (Baz \& García-Boyero, 1995, Wettstein \& Schmid, 1999, Steffan-Dewenter \& Tscharntke, 2000, Zschokke, et al., 2000, Krauss, et al., 2003) y otros grupos taxonómicos (Rosenzweig, 1995). En otros estudios se ha evidenciado que la densidad de insectos puede disminuir o aumentar con el incremento del área (Connor, et al., 2000, Matter, 2000, Steffan-Dewenter \& Tscharntke, 2000). En este estudio específico solo se pudo demostrar un aumento de la diversidad de mariposas con el incremento en el número de parches en el paisaje y la longitud de sus bordes y con la disminución del tamaño promedio de los parches, lo que significaría que en paisajes con un gran número de parches pequeños de formas muy irregulares, hay variedad de nichos que pueden servir como refugios para la lepidopterofauna. Por ello, se deduce que los fragmentos pequeños pueden ayudar a retener la diversidad de mariposas en paisajes tropicales e incrementar el valor de la conservación de los paisajes, como lo señala Pérez-García (2008).

Mediante el análisis de clases es posible cuantificar la configuración espacial de los tipos de hábitats que componen el mosaico de un paisaje, por lo que estas pueden utilizarse como una medida que refleja la fragmentación de cada tipo de hábitat y su grado de extensión (McGarigal \& Marks, 1994). En este sentido, se observó que los bosques de la cuenca del río Lagunillas se caracterizaban por ser un hábitat disminuido en cuanto al área y el número de fragmentos, y que su extensión era más significativa en zonas ubicadas entre los 1.100 y $1.700 \mathrm{~m}$ de altitud (Alto del Bledo y Palma Peñitas, respectivamente). No obstante, los valores más altos en cuanto al tamaño promedio de los parches se localizaron entre los 1.700 y $2.400 \mathrm{~m}$ y fueron menores en zonas bajas, dado que la irregularidad de su forma y su mayor longitud de borde determinaron una mayor heterogeneidad por debajo de esta última cota altitudinal (2.400 m), es decir, los bosques eran más regulares pero fragmentados en la localidad más alta (La Gloria, 2.950 m), y más pequeños pero muy irregulares y complejos por debajo de los 1.700 m. Saunders, et al. (1991) observaron que, aunque los remanentes más grandes de áreas naturales pueden mantener una mayor diversidad, un conjunto de fragmentos más pequeños pueden representar una mayor variedad de hábitats para una región y contribuir con una diversidad significativa.

La irregularidad de la forma y la complejidad de los fragmentos, vistas como una alteración o transformación que influye positivamente en el número de individuos $\mathrm{y}$ especies de mariposas (correlaciones positivas), denotan la presencia de un número significativo de microhábitats $\mathrm{y}$ nichos disponibles para estos organismos: las larvas y los adultos de las mariposas disponen así de un espectro amplio donde pueden desarrollarse y evitan la competencia intraespecífica dependiente de las preferencias de microhábitat, la capacidad de desplazamiento del adulto y la asociación con otras características como el mimetismo (Willmott \& Mallet, 2004). Resultados similares fueron registrados por Solarte-Cabrera (2005), quien observó que el bosque no intervenido (primario) tenía la menor riqueza de especies y el menor número de especies únicas, mientras que los bosques con algún grado de intervención (primario entresacado y secundario entresacado), presentaban la mayor riqueza y el mayor número de especies únicas. DeVries, et al. (1999) señalan la misma situación en un bosque pluvial en el Amazonas ecuatoriano, así como el efecto positivo en la riqueza de hábitats con nivel bajo o intermedio de perturbación, tal como se observó en este estudio.

Además, según Reinoso, et al. (2008b) la composición florística encontrada en los relictos de bosque de la cuenca del río Lagunillas fue de 980 especies, de las cuales 153 se encontraron en el bosque seco tropical (localidad de El Chorrillo), 212 en bosque húmedo premontano (localidad de Alto del Bledo), 175 y 238 en bosque húmedo montano bajo (localidades de Palma Peñitas y El Recodo respectivamente) y 202 en bosque húmedo montano (localidad de La Gloria). Además, los autores reiteran que teniendo en cuenta la riqueza y los índices ecológicos que utilizaron, asumieron que los bosques evaluados poseían distintos estadios de sucesión y de conservación que incluían desde sistemas con alteraciones marcadas y estadios tempranos de sucesión, como aquellos encontrados en el bosque seco tropical y en el premontano, hasta sistemas conservados y en estadios de sucesión más avanzados, como los encontrados en los demás bosques estudiados. Aunque fragmentados y con poca riqueza y diversidad de mariposas, estos son bosques conservados cuya principal especie arbórea es el roble y constituyen un museo al natural, ya que, según comentarios de pobladores de la región, algunos poseen árboles de más de 300 años, lo que confirma la importancia de este tipo de hábitat para la conservación de mariposas en paisajes agrícolas, tal como lo han demostrado diversos estudios (Ricketts, et al., 2001, Horner-Devine, et al., 2003, Harvey, et al., 2006, TobarLópez, et al., 2007).

El hábitat de matorrales o rastrojos fue el más representativo en el paisaje, con un número de parches mayor que los demás hábitats estudiados y con variaciones significativas entre las localidades (ventanas); sin embargo, aunque la extensión de este hábitat entre ventanas era similar (excepto en La Gloria), se observó una disminución en el número de parches a medida que se ascendía en el gradiente altitudinal; además, la longitud del borde evidenciaba formas irregulares en las ventanas de mayor altitud, lo que sugiere una mayor fragmentación en estas zonas. Aunque no se encontraron correlaciones significativas entre las variables de paisaje y la riqueza y diversidad de mariposas, en términos generales se 
podría plantear que las formas regulares (baja complejidad de forma) resultarían en un mayor número de individuos y de especies. Dennis, et al. (2007) han observado que la actividad de las mariposas es significativamente menor en matorrales, rastrojos y arbustos (perchan, descansan, toman el sol, duermen), que en zonas abiertas (pastizales), y que el número de individuos y especies aumenta con el incremento en el tamaño de los parches.

Por otra parte, los pastos constituían el hábitat con mayor extensión en el paisaje y las métricas evidenciaron que entre más extensos mayor era el número de individuos y menor la diversidad debido a la dominancia de algunas especies del grupo. Sin embargo, la diversidad puede verse favorecida cuando la irregularidad de forma aumenta. Aspectos como la exposición lumínica condicionan la presencia de ciertas especies por su relación con los hábitos alimenticios. Así, el grado de insolación en este tipo de hábitat determinaría la presencia del recurso floral para los adultos y de plantas nutricias para los estadios inmaduros (Pollard \& Yates, 1993). Cabe señalar que la exposición lumínica en estos sitios se da durante todo el día, aspecto que ha sido observado igualmente por Kremen (1992), quien encontró que las comunidades de mariposas están muy correlacionadas con la diversidad de plantas con flor y que, por el contrario, en el interior de las formaciones boscosas el grado de insolación es un factor crítico que influye en la selección del hábitat de muchas especies de mariposas. Además, en los pastizales los cambios en la composición y la alteración del crecimiento y de la diversidad vegetal ocasionada por la ganadería intensiva (Krues \& Tscharnke, 2002), hacen que los parámetros microclimáticos (temperatura, humedad, radiación solar) sean más intensos en estos hábitats e incidan en la distribución, la composición, la abundancia y la riqueza de especies de mariposas (Collinge, et al., 2003).

A diferencia de otros sistemas, los pastizales logran mantener una riqueza y abundancia de especies significativas en el paisaje fragmentado, ya que pueden conservar una variedad de especies más efectiva dependiendo de la composición de las especies vegetales y del arreglo espacial del paisaje. Si estos sistemas se encuentran en cercanías del bosque o entre fragmentos de este, permiten una conectividad estructural en el paisaje y son eficaces en la conservación de la biodiversidad (Harvey, et al., 2000, 2006).

\section{Conclusiones}

Los paisajes más fragmentados y complejos pueden ser más ricos y diversos que aquellos con características estructurales menos complejas y homogéneas; las diferencias estructurales y algunas características propias de cada hábitat pueden generar cambios en la riqueza y la diversidad; algunas características como el tamaño y el número de parches, la complejidad de su forma y su variabilidad, pueden limitar la cantidad de individuos y especies. La abundancia y la riqueza de las mariposas dependen del contexto del paisaje local.

\section{Conflicto de intereses}

Los autores declaran que no tienen conflicto de intereses.

\section{Bibliografía}

Álvarez, J. (1993). Inventario de las mariposas (Lepidoptera: Rhopalocera) con anotaciones ecológicas para dos zonas del departamento de Risaralda, Colombia. Trabajo de grado (Biología). Facultad de Ciencias, Universidad Nacional, Bogotá. 204 p.

Andrade-C, M.G. (1990). Clave para las familias y subfamilias de Lepidoptera: Rhopalocera de Colombia. Caldasia. 16 (79): 359-550.

Andrade-C, M.G. (1995). Monografías de fauna en Colombia 1. Nymphalidae: Acraeinae: Actinote. (Monografía No. 1). Instituto de Ciencias Naturales. Universidad Nacional de Colombia, Bogotá, 120 p.

Andrade-C., M.G. (2002). Biodiversidad de las mariposas (Lepidoptera: Rhopalocera) de Colombia. En: Costa, C., Vanin, S. A., Lobo, J. M., Melic, A. (editores.). Proyecto de Red Iberoamericana de Biogeografía y Entomología Sistemática - PriBES II. Monografías Tercer Milenio. Zaragoza: SEA. 2: 153-172.

Andrade-C., M.G. (2011). Estado del conocimiento de la biodiversidad en Colombia y sus amenazas. Consideraciones para fortalecer la interacción ambiente-política. Revista de la Academia Colombiana de Ciencias Exactas, Físicas y Naturales. 35 (137): 491-507.

Andrade-C., M.G., Campos-Salazar, L.R., González-Montaña, L.A., Pulido-B., H.W. (2007). Santa María, mariposas, alas y color. Serie de Guías de campo del Instituto de Ciencias Naturales No. 2. Instituto de Ciencias Naturales, Universidad Nacional de Colombia, Bogotá D. C., Colombia. 248 p.

Andrade-C., M.G., Henao-Bañol, E.R., Triviño, P. (2013). Técnicas y procesamiento para la recolección, preservación y montaje de mariposas en estudios de biodiversidad y conservación (Lepidoptera: Hesperioidea - Papilionoidea). Revista de la Academia Colombiana de Ciencias Exactas, Físicas y Naturales. 37 (144): 311-325.

Bastidas, A., Rivadeneira, P., Castillo, G. (2002). Variación de la diversidad de Lepidoptera Rhopalocera según gradientes altitudinales en el santuario de flora y fauna Galera (NariñoColombia). Resumen. XXXVII Congreso Nacional de Ciencias Biológicas. p. 241.

Baz, A. \& García-Boyero, A. (1995). The effects of forest fragmentation on butterfly communities in central Spain. Journal of Biogeography. 22: 129-140.

Birket, S.J. (1959). Genital preparations of male Lepidoptera. Entomologiske Meddeleser. 29: 170-178.

Blackburn, T.M. \& Gaston, K.J. (1996). Spatial patterns in the geographic range sizes of bird species in the New World. Philosophical Transactions of the Royal Society Biological Sciences. 351: 897-912.

Blue Marble Geographics. (1992-1994). The Geographic Calculator - version 3.09. 
Brosi, B.J., Shih, T.M., Billadello, L.N. (2008). Polinización biótica y cambios en el uso de la tierra en paisajes dominados por humanos. In C. Harvey \& J. C. Sáenz (editores). Evaluación y conservación de biodiversidad en paisajes fragmentados de Mesoamérica. Santo Domingo de Heredia, Costa Rica: Instituto Nacional de Biodiversidad INBio. p. 105-135.

Brown, K.S. (1991). The conservation of neotropical environments: Insects as indicator. In: The conservation of insects and their habits. Collins, N.M y J.A. Thomas, editors. Academic Press. p. 449 - 504.

Collinge, S.K., Prudic, K.L., Oliver, J.C. (2003). Effects of local habitat characteristics and landscape context on grassland butterfly diversity. Conservation Biology. 17 (1): 178-187.

Colwell, R.K. \& Hurtt, G.C. (1994). Nonbiological gradients in species richness and spurious Rapoport effect. American Naturalist. 144: 570-595.

Constantino, L.M. (1995). Revisión de la tribu Haeterini Herrich - Schaeffer, 1864 en Colombia (Lepidoptera: Nymphalidae: Satyrinae). SHILAP Revista Lepidopterológica. 23 (89): 49-76.

Connor, E.F., Courtney, A.C., Yoder, J. M. (2000). Individualsarea relationships: The relationship between animal population density and area. Ecology. 81: 734-748.

D’Abrera, B. (1981). Butterflies of the Neotropical Region. Part I. Papilionidae and Pieridae. Melbourne, Australia: Landsdowne Editions. XVI + 172 p.

D’Abrera, B. (1984). Butterflies of the neotropical region. Part II. Danaidae, Ithomiidae, Heliconidae \& Morphidae. Melbourne, Australia: Hill House Publishers. XII + 173384 p.

D’Abrera, B. (1987a). Butterflies of the neotropical region. Part III. Brassolidae, Acraeidae, Nymphalidae (partim). Melbourne, Australia: Hill House Publishers. VIII + 385-525 p.

D’Abrera, B. (1987b). Butterflies of the neotropical region. Part IV. Nymphalidae (partim). Melbourne, Australia: Hill House Publishers. XIV + 527-678 p.

D’Abrera, B. (1988). Butterflies of the neotropical region. Part V. Nymphalidae (Conc.) and Satyridae. Melbourne, Australia: Hill House Publishers. VIII + 679-877 p.

D’Abrera, B. (1994). Butterflies of the neotropical region. Part VI. Riodinidae. Melbourne, Australia: Hill House Publishers. VII + 879-1096 p.

D’Abrera, B. (1995). Butterflies of the neotropical region. Part VII. Lycaenidae. Melbourne, Australia: Hill House Publishers. XI + 1098-1270 p.

De La Maza, R.R. (1987). Mariposas mexicanas. México: Fondo de cultura Económica. 301 p.

Dennis, R., Shreeve, T.G., Sheppard, D A. (2007). Species conservation and landscape management: A habitat perspective. In A. Stewart, T. R. New, O. Lewis (editors). Insect Conservation Biology. CABI. p. 92-126.

DeVries, P.J. (1987). Butterflies of Costa Rica and their natural history: Papilionidae, Pieridae, Nymphalidae. New Jersey: Princeton. Vol. 1. 329 p.
DeVries, P.J., Murray, D., Lande, R. (1997). Species diversity in vertical, horizontal, and temporal dimensions of a fruitfeeding butterfly community in an Ecuadorian rainforest. Biological Journal of the Linnean Society. 62: 343-364.

DeVries, P.J., Walla, T., Greeney, H. (1999). Species diversity in spatial and temporal dimensions of fruit-feeding butterflies from two Ecuadorian rainforests. Biological Journal of the Linnean Society. 68: 333-353.

Didham, R.K., Lawton, J.H., Hammond, P.M., Eggleton, P. (1998). Trophic structure stability and extinction dynamics of beetles (Coleoptera) in tropical forest fragments. Philosophical Transactions of the Royal Society Biological Sciences. 353: 437-451.

Ekroos, J. \& Kuussaari, M. (2012). Landscape context affects the relationship between local and landscape species richness of butterflies in semi-natural habitats. Ecography. 35: 232238.

Erelli, M.C., Ayres, M.P., Eaton, G.K. (1998). Altitudinal patterns in host suitability for forest insects. Oecology. 117: 133-142.

Etter, A. (1993). Ecosystem diversity in Colombia. In: Our Biological Diversity, CEREC and A. Angel Foundation (editors). Bogotá. p. 43-61.

Etter, A. \& van Wyngaarden, W. (2000). Patterns of landscape transformation in Colombia, with emphasis in the Andean region. Royal Swedish Academy of Sciences, Ambio. 29 (7): 432-439.

Evans, W.H. (1951). A catalogue of the American Hesperiidae indicating the classification and nomenclature adopted in the British Museum (Natural History). Part I. Introduction and Group A Pyrrhopyginae. London, British Museum (Natural History). 92 p.

Evans, W.H. (1952). A catalogue of the American Hesperiidae indicating the classification and nomenclature adopted in the British Museum (Natural History). Part II. Pyrginae. Section I. London, British Museum (Natural History). 178 p.

Evans, W.H. (1953). A catalogue of the American Hesperiidae indicating the classification and nomenclature adopted in the British Museum (Natural History). Part III. Pyrginae. Section 2. London, British Museum (Natural History). 246 p.

Evans, W.H. (1955). A catalogue of the American Hesperiidae indicating the classification and nomenclature adopted in the British Museum (Natural History). Part IV. Hesperiinae and Megathyminae. London, British Museum (Natural History). 499 p.

Fagua, G. (1999). Variación de las mariposas y hormigas de un gradiente altitudinal de la cordillera Oriental (Colombia). Revista Insectos de Colombia. 2: 318-363.

Fagua, G. (2001). Mariposas diurnas (Lepidoptera). Manual de metodologías para el desarrollo de inventarios y monitoreo de la biodiversidad. Instituto de Investigación de Recursos Biológicos Alexander von Humboldt. Grupo de Exploración y Monitoreo ambiental - GEMA. p. 59 - 76.

Fleishman, E., Austin, E., Weiss, A.D. (1998). An empirical test of Rapoport's rule: Elevational gradients in montane butterfly communities. Ecology. 79 (7): 2482-2493. 
Fox, M. \& Real, H.G. (1971). A Monograph of the Ithomiidae (Lepidoptera). Part IV. The tribe Napeogenini Fox. Memoirs of the American Entomological Institute. 15: 368.

García-Pérez, J.F. \& Ospina-López, L.A. (2004). Lepidoptera: Rhopalocera: diversidad y distribución en la cuenca del río Coello. Ibagué. Tesis de grado. Programa de Biología, Facultad de Ciencias Básicas, Universidad del Tolima. 485 p.

García-Pérez, J.F., Ospina-López, L.A., Villa, F.A., Reinoso, G. (2007). Diversidad y distribución de mariposas Satyrinae (Lepidoptera: Nymphalidae) en la cuenca del río Coello, Colombia. Revista de Biología Tropical. 55: 645-653.

García-Robledo, C., Constantino, L.M., Dolores, M., Kattan, G. (2002). Mariposas comunes de la cordillera Central de Colombia. Programa de Colombia de Wildlife Conservation Society. Cali: Feriva, S.A. 130 p.

Gascon, C., Lovejoy, T.E., Bierregaard, R.O., Malcolm, J.R., Stou, P.C., Vasconcelos, H.L., Laurance, W.F. (1999). Matrix habitat and species richness in tropical forest remnants. Biological Conservation. 91: 223-229.

Hammer, Ø., Harper, D.A., Ryan, P.D. (2001). PAST: Paleontological Statistics Software Package for Education and Data Analysis. Paleontología Electrónica. 4 (1): 1-9.

Harvey, C.A., Guindon, C.F., Haber, W.A., Hamilton-Derosier, D., Murray, K.G. (2000). The importance of forest patches isolated trees and agricultural windbreaks for local and regional biodiversity: The case of Monteverde. Costa Rica. In: XXI IUFRO World Congress 7-12 August 2000. Kuala Lumpur. Malasya. Subplenary Sessions. Vol I. XXI IUFRO World Congress. International Union of Research Organization. Kuala Lumpur, Malasya. p. 787-798.

Harvey, C.A., Medina, A., Merlo-Sánchez, D., Vílchez, S., Hernández, B., Sáenz, J.C., Maes, J.M., Casanoves, F., Sinclair, F.L. (2006). Patterns of animal diversity in different forms of tree cover in agricultural landscapes. Ecological Applications. 16: 261-289.

Hill, J.K., Hamer, K.C., Dawood, M.M., Tangah, J., Dawood, D. (2001). Ecology of tropical butterflies in rainforest gaps. Oecologia. 128: 294-302.

Horner-Devine, M.C., Daily, G.C., Ehrlich, P.R. (2003). Countryside biogeography of tropical butterflies. Conservation Biology. 17 (1): 168-177.

Jeanneret, P.H., Schüpbach, B., Luka, H. (2003). Quantifying the impact of landscape and habitat features on biodiversity in cultivated landscapes. Agriculture Ecosystems and Environment. 98: 311-320.

Kattan, G. (2002). Fragmentación: patrones y mecanismos de extinción de especies. En M. R. Guariguata \& G. Kattan (editores). Ecología y Conservación de Bosques Neotropicales. Cartago, Costa Rica: Libro Universitario Regional. p. 561-590.

Krauss, J., Steffan-Dewenter, I., Tscharntke, T. (2003). How does landscape context contribute to effects of habitat fragmentation on diversity and population density of butterflies? Journal of Biogeography. 30: 889-900.
Kremen, C. (1992). Assessing the indicator properties of species assemblages for natural areas monitoring. Ecological Applications. 2 (2): 203-217.

Kremen, C., Colwell, R.K., Erwin, T.L., Murphy, D.D., Noss, R.F., Sanjayan, M.A. (1993). Terrestrial arthropod assemblages: Their use in conservation planning. Conservation Biology. 7 (4): 796-808.

Krues, A. \& Tscharntke, T. (2002). Grazing intensity and the diversity of grasshoppers, butterflies and trap-nesting bees and wasps. Conservation Biology. 16 (6): 1570-1580.

Kuussaari, M., Bommarco, R., Heikkinen, R.K., Helm, A., Krauss, J., Linborg, R. (2009). Extinction debt: A challenge for biodiversity conservation. Trends in Ecology \& Evolution. 24: 564-571.

Lamas, G. (2003). Estado actual del conocimiento de la sistemática de los lepidópteros, con especial referencia a la región Neotropical. Boletín de la Sociedad Entomológica Aragonesa. No. 32.

Lamas, G. (2004). Atlas of Neotropical Lepidoptera. Checklis: Part 4: Hesperioidae-Papilionoidea. Florida (USA): Association for Tropical Lepidoptera. 439 p.

Lande, R. (1996). Statistics and partitioning of species diversity, and similarity among multiple communities. Oikos. 76: 5-13.

Le Crom, J.F., Constantino, L.M., Salazar, J.A. (2002). Mariposas de Colombia. Tomo I: Papilionidae. Bogotá: Carlec Ltda. 119 p.

Le Crom, J.F., Llorente, J., Constantino, L.M., Salazar, J.A. (2004). Mariposas de Colombia. Tomo II: Pieridae. Carlec Ltda., Bogotá. 133 p.

Magurran, A.E. (1988). Ecological diversity and its measurement. New Jersey: Princeton University Press.

Magurran, A.E. (2004). Measuring biological diversity. USA: Blackwell Publishing. Vol VIII. 256 p.

Matter, S.F. (2000). The importance of the relationship between population density and habitat area. Oikos. 89: 613-619.

McGarigal, K. \& Marks, B.J. (1994). FRAGSTATS: Spatial pattern analysis program for quantifying landscape structure. Corvallis, Oregon. 134 p.

McGarigal, K., Cushman, S.A., Neel, M.C., Ene, E. (2002). FRAGSTATS v3: Spatial Pattern Analysis Program for Categorical Maps. Computer software program produced by the authors at the University of Massachusetts, Amherst. www.umass.edu/landeco/research/fragstats/fragstats.html

Moreno, C.E. (2001). Métodos para medir la biodiversidad. M\&TManuales y Tesis. Zaragoza: SEA. Vol. 1., 84 p.

Murcia, C. (1995). Edge effects in fragmented forests: Implications for conservation. Trends in Ecology and Evolution. 10: $58-62$.

Murdoch, W.W., Evans, F.C., Peterson, C.H. (1972). Diversity and pattern in plants and insects. Ecology. 53: 819-829.

Neild, A.F.E. (1996). The butterflies of Venezuela, Part 1: Nymphalidae I (Limenitidinae, Apaturinae, Charaxinae). London: Meridian Publications. 144 p. 
Odum, E. (1971). Fundamentals of Ecology. W. B. Saunders Company. p. 574.

Ospina-López, L. A., García-Pérez, J.F., Villa, F., Reinoso, G. (2010). Mariposas Pieridae (Lepidoptera: Papilionoidea) de la cuenca del rio Coello, Tolima, Colombia. Actualidades Biológicas. 32 (93): 173-188.

Peña-Cerpa, J.M. (2007). Diversidad y distribución de mariposas diurnas (Lepidoptera: Rhopalocera) en la cuenca del río Prado. Tesis de grado. Programa de Biología, Facultad de Ciencias Básicas, Universidad del Tolima, Ibagué. 180 p.

Pérez-García, O. (2008). Evaluación de la biodiversidad de mariposas diurnas presentes en sistemas agroforestales modernos con café en el Corredor Biológico Volcánica Central-Talamanca, Costa Rica. Tesis Magister Scientiae; Escuela de posgrado; Centro Agronómico Tropical de Investigación y Enseñanza. Turrialba, Costa Rica. 80 p.

Pollard, E. \& Yates, T.J. (1993). Monitoring butterflies for ecology and conservation. Londres: Chapman y Hall. 277 p.

Reinoso, G., Villa, F., Esquivel, H.E., García-Melo, J.E., Vejarano-Delgado, M.A. (2007). Biodiversidad faunística y florística de la cuenca del río Totare - Biodiversidad regional Fase III. Informe Técnico. Cortolima - Universidad del Tolima. $1.231 \mathrm{p}$.

Reinoso, G., Villa, F., Esquivel, H.E., García-Melo, J.E., Vejarano-Delgado, M.A. (2008a). Biodiversidad faunística y florística de la cuenca del río Saldañasubcuenca Anamichú - Biodiversidad regional Fase IV. Informe Técnico. Cortolima - Universidad del Tolima. 943 p.

Reinoso, G., Villa, F., Esquivel, H.E., García-Melo, J.E., Vejarano-Delgado, M.A. (2008b). Biodiversidad faunística y florística de la cuenca del río Lagunillas - Biodiversidad regional Fase IV. Informe Técnico. Cortolima - Universidad del Tolima. 965 p.

Rempel, R.S., Kaukinen, D., Carr, A.P. (2012). Patch Analyst and Patch Grid. Ontario Ministry of Natural Resources. Centre for Northern Forest Ecosystem Research, Thunder Bay, Ontario.

Ricketts, T.H., Daily, G.C., Ehrlich, P.R., Fay, J.P. (2001). Countryside biogeography of moths in a fragmented landscape: Biodiversity in native and agricultural habitats. Conservation Biology. 15 (2): 378-388.

Rosenzweig, M.L. (1995). Species diversity in space and time. Cambridge, UK: Cambrige University Press. 460 p.

Sanders, N.J. (2002). Elevational gradients in ant species richness: Area, geometry and Rapoport's rule. Ecography. 25: 25-35.

Saunders, D.A., Hobbs, R.J., Margules, C.R. (1991). Biological Consequences of Ecosystem Fragmentation: A Review. Conservation Biology. 5 (1): 18-32.

Schulze, C.H., Waltert, M., Kessler, P.J., Pitopang, R., Shahabuddin, Veddeler, D., Mühlenberg, M., SteffanDewenter, I., Gradstein, S.R., Tscharntke, T. (2004). Biodiversity indicator taxa of tropical land-use systems: Comparing plants, birds and insects. Ecological Application. 14 (5): 1321-1333.
Seitz, A. (1924). The macrolepidoptera of the world. Alfred Kernen Verlag Stuttgart. Vol. V. 1139 p.

Simonson, S.E., Opler, P.A., Stohlgren, T.J., Chong, G.W. (2001). Rapid assessment of butterfly diversity in a montane landscape. Biodiversity Conservation. 10: 1369-1386.

Sokal, R. \& Rohlf, J. (1995). Biometry. The principles and practice of statistics in biological research. 3rd Ed. W. H. New York: Freeman and Company. 850 p.

Solarte-Cabrera, V.M. (2005). Diversidad y estructura espaciotemporal de la comunidad de mariposas diurnas en la Reserva Natural Río Ñambí. San Juan de Pasto. Trabajo de grado. Programa de Biología, Facultad de Ciencias Naturales y Matemáticas, Universidad de Nariño. p. 102.

Southwood, T.R., Brown, V.K., Reader, P.M. (1979). The relationship of plant and insect diversities in succession. Zoological Journal of Linnean Society. 12: 327-348.

Statsoft, I. (2007). STATISTICA (data analysis software system). www.statsoft.com

Steffan-Dewenter, I. \& Tscharntke, T. (2000). Butterfly community structure in fragmented habitats. Ecology Letters. 3: 449-456.

Stevens, G.C. (1992). The elevational gradient in altitudinal range: An extension of Rapoport's latitudinal rule to altitude. American Naturalist. 140: 893-911.

Tobar-López, D., Muhammad, I., Casasola, F. (2007). Diversidad de mariposas en un paisaje agropecuario en la región Pacifico Central, Costa Rica. Agroforestería de las Americas. 47: 58-65.

Tyler, H., Brown, K., Wilson, K. (1994). Swallowtail Butterflies of the Americas. A study in biological dynamics, ecological diversity, biosystematics and conservation. Gainesville, US: Scientific Publishers Inc. 376 p.

Vargas, R., Rojas, A.M., Huertas, F.A., Fernández, D.L., Murillo, M.H., Dávila, Y.P., Valderrama, S. (2007). Plan General de Ordenación Forestal para el Departamento del Tolima, Tomo II. Ibagué, Colombia: Universidad del Tolima. 403 p.

Villa, F., Reinoso, G., Bernal, M.H., Losada, S. (2004). Biodiversidad faunística de la cuenca del río Coello Biodiversidad regional Fase I. Informe Técnico. Cortolima - Universidad del Tolima. 1132 p.

Villa, F., Reinoso, G., Losada, S., Bernal, M.E., Esquivel, H.E., García-Melo, J.E., Vejarano-Delgado, M.A. (2005a). Biodiversidad faunística y florística de la cuenca del río Prado-Biodiversidad regional Fase II. Informe Técnico. Cortolima - Universidad del Tolima. 1308 p.

Villa, F., Reinoso, G., Losada, S., García-Melo, J.E., VejaranoDelgado, M.A. (2005b). Biodiversidad faunística y florística de la cuenca del río Amoyá - Biodiversidad regional Fase II. Informe Técnico. Cortolima - Universidad del Tolima. $823 \mathrm{p}$.

Vu, L. (2009). Diversity and similarity of butterfly communities in five different habitat types at Tam Dao National Park, Vietnam. Journal of Zoology. 277 (1): 15-22. 
Warren, A.D., Davis, K.J., Stangeland, E.M., Pelham, J.P., Grishin, N.V. (2013). Illustrated Lists of American Butterflies. [30-XII-2013]. < http://www. butterfliesofamerica.com/ >

Wettstein, W. \& Schmid, B. (1999). Conservation of arthropod diversity in montane wetlands: Effect of altitude, habitat quality and habitat fragmentation on butterflies and grasshoppers. Journal of Applied Ecology. 36: 363-373.

Willmott, K. (2003). The genus Adelpha: Its systematics, biology, and biogeography. Gainesville, USA: Scientific Publishers. 322 p.
Willmott, K. \& Mallet, J. (2004). Correlations between adult mimicry and larval host plants in Ithomiine butterflies. Proceedings of the Royal Society B. 271 (Suppl.): 266-269.

Zschokke, S., Dolt, C., Rusterholz, H., Oggier, P., Braschler, B., Thommen, G.H., Lüdin, E., Erhardt, A., Baur, B. (2000). Short-term responses of plants and invertebrates to experimental small-scale grassland fragmentation. Oecologia. 125: 559-572. 